\title{
SINGULARITIES OF VARIATIONS OF MIXED HODGE STRUCTURE*
}

\author{
AROLDO KAPLAN ${ }^{\dagger}$ GREGORY PEARLSTEIN ${ }^{\ddagger}$
}

\begin{abstract}
We give a condition for a variation of mixed Hodge structure on a curve to be admissible at a singular point - hence to have a logarithmic singularity there - and describe the monodromy, all in terms of a special variable grading of the weight filtration. The result exactly supplements the information about the graded variation implied by Schmid's Orbit Theorems. The grading is relevant for the irregular case as well.
\end{abstract}

1. Introduction. Let $f: X \rightarrow \Delta$ be a semistable degeneration over the unit disk in $\mathbb{C}$. As a consequence of Schmid's nilpotent orbit theorem, the period map of the associated variation of pure Hodge structure $\mathcal{V}=R_{f *}^{n}(\mathbb{Q}) \otimes \mathcal{O}_{\Delta^{*}}$ has at worst logarithmic singularities at the origin [12][20]. More generally, given any abstract variation of Hodge structure $\mathcal{V} \rightarrow \Delta^{*}$ with unipotent monodromy, Schmid's theorem implies that the corresponding period map has at worst logarithmic singularities at the origin.

Replacing the semistable degeneration $f$ by an arbitrary morphism leads to the notion of variation of mixed Hodge structure. As in the pure case, such a variation $\mathcal{V} \rightarrow \Delta^{*}$ is described by a period map

$$
\varphi: \Delta^{*} \rightarrow \mathcal{M} / \Gamma
$$

which takes values in a moduli space of graded-polarized mixed Hodge structure. Unlike the pure case, however, the period map of an abstract variation of mixed Hodge structure can have irregular singularities at zero.

The only bound upon the possible singularities of $\varphi$ is the polarization of the pure structures on the graded pieces of the weight filtration $\mathcal{W}$ of $\mathcal{V}$ : the nilpotent orbit theorem implies that the period map of the graded variation $G r^{\mathcal{W}}(\mathcal{V})$ has at worst logarithmic singularities. Therefore, all of the irregularities of $\varphi$ must come from the extension data $G r^{\mathcal{W}}(\mathcal{V}) \mapsto \mathcal{V}$.

There are many ways of codifying this data, e.g. many gradings of the weight filtration $\mathcal{W}$ compatible with the Hodge filtration $\mathcal{F}$ of $\mathcal{V}$. In this article we show that, among these, the grading

$$
\mathcal{E}_{k}=\bigoplus_{p+q=k} \mathcal{I}^{p, q}
$$

defined by the Deligne-Hodge factors $\mathcal{I}^{p, q}$ is quite appropriate to study the singularity.

Suppose, for example, that $\mathcal{E}$ extends continuously to the canonical extension $\tilde{\mathcal{V}} \rightarrow \Delta$ of $\mathcal{V}[11]$. Let $\rho$ be the unique representation of $s_{2}(\mathbb{C})$ on $V:=\tilde{\mathcal{V}}(0)$ that

* Received January 24, 2003; accepted for publication July 2, 2003. The authors 'wish to thank P. Deligne, whose ideas play an important role here, as well as the referee for valuable suggestions about the exposition.

$\dagger$ Department of Mathematics, University of Massachusetts, Amherst, MA 01003, USA and Centro de Investigaciones y Estudios Matemáticos, Universidad de Córdoba, Ciudad Universitaria, 5000 Cordoba, Argentina (kaplan@math.umass.edu).

$\ddagger$ Department of Mathematics, University of California, Irvine, CA 92697-3875, USA (gpearlst@math.uci.edu). 
leaves $E=\mathcal{E}(0)$ invariant and induces Schmid'd limiting $s l_{2}$-representations in the graded quotients (cf. $\S 2)$. Let

$$
N=N_{0}+N_{-1}+N_{-2}+\cdots
$$

denote the decomposition of the monodromy logarithm with respect to grading $E$. We will prove that, under the conditions of Theorems I-III below,

(a) $N_{0}=\rho\left(\begin{array}{ll}0 & 0 \\ 1 & 0\end{array}\right)$; $k-2$.

(b) For $k>0, N_{-k}$ is either zero, or a highest weight vector for ad $\rho$ of weight

In particular, $N_{-1}=0$. These conditions provide a complete description of the possible monodromy of such a variation, generalizing another of Schmid's theorems to the mixed case.

To compare our result with the existing literature, we recall the following definition of Steenbrink and Zucker [23]:

Definition. A variation of graded-polarized mixed Hodge structure $\mathcal{V} \rightarrow \Delta^{*}$ with unipotent monodromy is admissible if and only if

(1) The Hodge filtration $\mathcal{F}$ extends to $\tilde{\mathcal{V}}$, and induces the limiting Hodge filtration of Schmid on $G r^{\mathcal{W}} \tilde{\mathcal{V}}$;

(2) The relative weight filtration ${ }^{r} W={ }^{r} W(N, \mathcal{W})$ exists.

The existence of the limiting grading $E$ implies that $\mathcal{V}$ is admissible. (1) follows from the nilpotent orbit theorem while (2) follow from (a),(b). Indeed, let $Y$ be the endomorphism of $V$ which acts as multiplication by $k$ on $E_{k}$. Then,

$$
{ }^{r} Y=Y+\rho\left(\begin{array}{cc}
1 & 0 \\
0 & -1
\end{array}\right)
$$

is a semisimple endomorphism whose eigenspaces grade ${ }^{r} W$. Moreover, the above formula for ${ }^{r} Y$ implies that in this case, the relative weight filtration equals the convolution of the weight filtration of $\mathcal{V}$ with the increasing filtration defined by the semisimple endomorphism

$$
H=\rho\left(\begin{array}{cc}
1 & 0 \\
0 & -1
\end{array}\right)
$$

Admissibility is a tameness condition on the variation; this is obvious in regard to the existence of $F$. As far as that of ${ }^{r} W$, this was so by analogy with the $\ell$-adic case [10]. Here we will see that ${ }^{r} W$ becomes an actual limit of natural filtrations of $\mathcal{V}$, filtrations that make sense even if the limit does not exist.

Every geometric variation - of the form $R_{f *}^{n}(\mathbb{Q}) \otimes \mathcal{O}_{\Delta^{*}}$, is admissible. This was proven by El Zein et. al. (1980's [4] §7) by extending Steenbrink's results on geometric variations of pure structures [22] and can be regarded as the effect of having some degenerated object at $s=0$. For an abstract variation of pure structures instead, the existence (and weighting) of the limiting Hodge filtration is major consequence of the nilpotent orbit theorem. This shows an obvious drawback of the notion of admissibility, namely that its use is restricted to situations where the conclusions of the orbit theorems are known to hold for independent reasons. Theorems I-III below can be regarded as a test for admissibility, to supplement Schmid's theorems in situations where irregular singularities may in principle occur. 
In this regard, we note the renewed interest in irregularities of integrable connections coming from arithmetic and geometry (see [1][2][3][16][21] and references thereof). In particular, variations of Hodge structures are examples of Higgs fields, which represent non-abelian cohomology classes of varieties; unless the latter is smooth and compact, the corresponding Higgs fields may have irregular singularities at infinity [1][21]. Irregular Higgs fields also represent certain "wild" enlargements of $\pi_{1}$ [2][15]; the analog for the latter of Hain's variations on fundamental groups should give further examples of non-admissible variations.

The analogy with tamely/wildly ramified covers in arithmetic is not coincidental and, in this regard, variations of mixed Hodge structure present an obvious kind of "simplest wild ramification", namely when the limiting Hodge filtration $F$ exists but the relative weight filtration ${ }^{r} W$ does not. A good part of our calculations remain valid in this case and a corresponding description of the ramification - what should replace ${ }^{r} W$ and how constrained the monodromy will be, is implicit in (4.8) below.

To properly state our results, recall that a mixed Hodge structure $(F, W)$ on a complex vector space $V=V_{\mathbb{R}} \otimes \mathbb{C}$ defines a unique bigrading

$$
V=\bigoplus_{p, q} I^{p, q}
$$

of $V$ with the following properties:

$$
\begin{gathered}
F^{p}=\oplus_{a \geq p, b} I^{a, b} \quad W_{k}=\oplus_{a+b \leq k} I^{a, b} \\
\bar{I}^{p, q} \equiv I^{q, p} \quad \bmod \bigoplus_{r<q, s<p} I^{r, s}
\end{gathered}
$$

In particular, a mixed Hodge structure determines a grading $Y_{(F, W)}$ of the underlying weight filtration $W$ by the rule

$$
Y_{(F, W)}(v)=k v \Longleftrightarrow v \in \bigoplus_{p+q=k} I^{p, q}
$$

This identification of a $\mathbb{Z}$-grading of $V$ with the semisimple endomorphism with degrees as eigenvalues, will be used throughout.

Our first result specifies the condition and asserts the equivalence in the case of variations that are unipotent in the sense of [13].

THEOREM I. Let $\mathcal{V} \rightarrow \Delta^{*}$ be a variation of graded-polarized mixed Hodge structure such that the graded variation is constant. Then, $\mathcal{V}$ is admissible if and only if the $C^{\infty}$-grading $\mathcal{Y}$ of $\mathcal{W}$ defined pointwise by (1.2) extends continuously to $\tilde{\mathcal{V}} \rightarrow \Delta$.

The implication .

$$
\mathcal{V} \text { admissible } \Longleftrightarrow \mathcal{Y} \text { extends }
$$

can be established in many other cases. Here, we will prove the full equivalence when the limiting objects are real in the sense specified below.

REMARK. It is not clear that the reality assumptions can be dropped altogether. One cannot just replace a variation of mixed structure by a split one, as one does in the pure case: in terms of Example (6.20) in [18], the monopole $h(y)$ becomes singular at infinity. 
Recall that a mixed Hodge structure $\left(F, W^{\prime}\right)$ is split over $\mathbb{R}$ if and only if

$$
\overline{I_{\left(F, W^{\prime}\right)}^{p, q}}=I_{\left(F, W^{\prime}\right)}^{q, p}
$$

Accordingly, we shall say that an admissible nilpotent orbit

$$
\left(e^{z N} \cdot F, W\right)
$$

of mixed Hodge structure is split if the mixed Hodge structure

$$
\left(F,{ }^{r} W(N, W)\right)
$$

splits over $\mathbb{R}$. Note that if a nilpotent orbit $\left(e^{z N} \cdot F, W\right)$ is admissible, that is precisely its limiting mixed Hodge structure. Our second result is:

THEOREM II. Let $\theta(z)=\left(e^{z N} \cdot F, W\right)$ be a nilpotent orbit of graded-polarized mixed Hodge structure such that the graded orbits are split. Then $\theta(z)$ is admissible and split if and only if the limit

$$
Y_{\infty}=\lim _{I m(z) \rightarrow \infty} \operatorname{Ad}\left(e^{-z N}\right) Y_{\theta(z)}
$$

exists and is defined over $\mathbb{R}$.

For a general variation, the limiting mixed Hodge structure is only defined once a coordinate on the base -more specifically, an element of $T_{0}(\Delta)^{*}$, has been chosen. We can then refer to split admissible variations, as those whose limiting mixed Hodge structure is split. With this understood, our third result is:

THEOREM III. Let $\mathcal{V} \rightarrow \Delta^{*}$ be a variation of graded-polarized mixed Hodge structure with unipotent monodromy, and $\mathcal{Y}$ denote the $C^{\infty}$ grading of the weight filtration $\mathcal{W}$ obtained by applying (1.2) to each fiber of $\mathcal{V}$. Suppose that the graded variations are split. Then, the variation $\mathcal{V}$ is admissible and split if and only if $\mathcal{Y}$ extends continuously to a grading of $\mathcal{W}$ in $\tilde{\mathcal{V}}$, with limiting value $\mathcal{Y}(0)=\lim _{s \rightarrow 0} \mathcal{Y}(s)$ defined over $\mathbb{R}$.

We now sketch the proofs of these results: Theorem I is proved in $\S 3$. One first writes the period map $F: U \rightarrow \mathcal{M}$ as

$$
F(z)=e^{z N} e^{\Gamma(s)} \cdot F_{\infty}
$$

where $F_{\infty}$ is the limiting Hodge filtration of $\mathcal{V}$, and $\Gamma(s)$ is a holomorphic function of $s=e^{2 \pi i z}$ which takes values in the nilpotent Lie algebra

$$
\operatorname{Lie}_{-1}=\left\{\alpha \in \operatorname{End}(V) \mid \alpha: W_{k} \rightarrow W_{k-1} \forall k\right\}
$$

and vanishes at $s=0$. To finish the proof we apply Theorem (2.9), which provides a description of how the decomposition $V=\oplus_{p, q} I^{p, q}$ varies under the action of $\exp \left(\right.$ Lie $\left._{-1}\right)$ on $\mathcal{M}$.

Theorems II and III are proved in $\S 4$ and $\S 5$, respectively. One of the implications in the first depends heavily upon the results of Deligne [8] discussed in the appendix at the end of our paper. They imply that if $W$ and $N$ arise from a nilpotent orbit of graded-polarized mixed Hodge structure $e^{z N} . F$ which is admissible and split, then

$$
Y_{\left(e^{z N} . F, W\right)}=A d\left(e^{z N}\right) \tilde{Y}
$$


where $\tilde{Y}$ is an $\mathbb{R}$-grading of $W$ which depends only on $N$ and ${ }^{r} Y=Y_{\left(F,{ }^{r} W\right)}$. In particular, whenever $e^{z N} . F$ is a split admissible nilpotent orbit, the associated grading

$$
\left.e^{-z N} \cdot Y_{\left(e^{z N}\right.} \cdot F, W\right)
$$

in Theorem II has constant value $Y_{\infty}=\tilde{Y}$. Likewise, if $\left(F,{ }^{r} W\right)$ is split over $\mathbb{R}$ then so are each of the induced mixed Hodge structures in the graded, and the corresponding nilpotent orbits are split.

To prove the converse, we recall that split nilpotent orbits of mixed Hodge structure are just $S L_{2}(\mathbb{R})$-orbits, assemble the corresponding representations in the graded into one on $V$ as already described, define

$$
{ }^{(r)} Y:=Y_{\infty}+\rho\left(\begin{array}{cc}
1 & 0 \\
0 & -1
\end{array}\right), \quad{ }^{(r)} W_{k}=\bigoplus_{j \leq k} E_{j}\left({ }^{(r)} Y\right)
$$

and prove that ${ }^{(r)} W_{k}$ is actually the relative weight filtration of $N$ and $W$. This is the most technically complicated part of the proof, involving the use of the $s l_{2}$ symmetry to deduce properties of certain Laurent series. Theorem III is a corollary of these proofs.

The significance of the grading $\mathcal{Y}$ was discussed in correspondence of Deligne with E. Cattani and one of us (Kaplan) dating back to 1992 [8]. Related results were presented in [6][14][17] and [18]. Some of the material from [8] and [14] is being published here for the first time.

2. Preliminaries. In this section we reformulate our theorems in terms of period maps, review the definition of admissibility, discuss the geometry of the associated classifying spaces and describe an analog of the Nilpotent Orbit Theorem for variations of graded-polarized mixed Hodge structure.

DEFinition. A variation of graded-polarized mixed Hodge structure consists of a $\mathbb{R}$-local system $\mathcal{V}_{\mathbb{R}} \rightarrow S$ endowed with the following additional data:

(1) A real, increasing weight filtration

$$
0 \subseteq \cdots \subseteq \mathcal{W}_{k-1} \subseteq \mathcal{W}_{k} \subseteq \cdots \subseteq \mathcal{V}_{\mathbb{C}}
$$

of $\mathcal{V}_{\mathbb{C}}=\mathcal{V}_{\mathbb{R}} \otimes \mathbb{C}$

(2) A decreasing Hodge filtration

$$
0 \subseteq \cdots \subseteq \mathcal{F}^{p} \subseteq \mathcal{F}^{p-1} \subseteq \cdots \subseteq \mathcal{V}_{\mathbb{C}} \otimes \mathcal{O}_{S}
$$

which is holomorphic and horizontal with respect to the Gauss-Manin connection $\nabla$ of $\mathcal{V}_{\mathbb{C}} \otimes \mathcal{O}_{S}$ (i.e. $\nabla \mathcal{F}^{p} \subseteq \Omega_{S}^{1} \otimes \mathcal{F}^{p-1}$ ) and pairs with the weight filtration $\mathcal{W}$ to define a mixed Hodge structure on each fiber of $\mathcal{V}$.

(3) A collection of real, non-degenerate bilinear forms

$$
\mathcal{S}_{k}: G r_{k}^{\mathcal{W}}\left(\mathcal{V}_{\mathbb{R}}\right) \otimes G r_{k}^{\mathcal{W}}\left(\mathcal{V}_{\mathbb{R}}\right) \rightarrow \mathbb{R}, \quad G r_{k}^{\mathcal{W}}:=\mathcal{W}_{k} / \mathcal{W}_{k-1}
$$

of alternating parity $(-1)^{k}$ which polarize the corresponding variations of pure Hodge structure

$$
\mathcal{F} G r_{k}^{\mathcal{W}}:=\frac{\mathcal{F}^{p} \cap \mathcal{W}_{k}+\mathcal{W}_{k-1}}{\mathcal{W}_{k-1}}
$$


The data of such a variation $\mathcal{V} \rightarrow S$ may be reformulated in terms of the monodromy representation [17]

$$
\rho: \pi_{1}\left(S, s_{o}\right) \rightarrow \operatorname{Aut}\left(\mathcal{V}_{s_{o}}\right), \quad \operatorname{Image}(\rho)=\Gamma
$$

of $\mathcal{V}$ on a given fiber $\mathcal{V}_{s_{o}}$ and the corresponding period map

$$
\varphi: S \rightarrow \mathcal{M} / \Gamma
$$

obtained by parallel translating the data of $\mathcal{V}$ to $\mathcal{V}_{s_{o}}$. Alternatively, upon passage to the universal cover $\tilde{S}$ of $S$, such a variation is equivalent to the monodromy representation $\rho$ defined above together with a $\pi_{1}$-equivariant map

$$
F: \tilde{S} \rightarrow \mathcal{M}
$$

from $\tilde{S}$ into a suitable classifying space $\mathcal{M}$ of graded-polarized mixed Hodge structure. $F$ is both holomorphic and horizontal: i.e.

$$
\frac{\partial}{\partial \bar{z}_{j}} F^{p}(z) \subseteq F^{p}(z), \quad \frac{\partial}{\partial z_{j}} F^{p}(z) \subseteq F^{p-1}(z)
$$

In particular, if $\mathcal{V} \rightarrow \Delta^{*}$ is a variation of graded-polarized mixed Hodge structure with unipotent monodromy action $T=e^{-N}$, its period map $\varphi$ may be viewed as a holomorphic, horizontal map

$$
F: U \rightarrow \mathcal{M}
$$

from the upper half-plane $U$ into $\mathcal{M}$ which satisfies the quasi-periodicity condition

$$
F(z+1)=e^{N} \cdot F(z) .
$$

Our choice of sign for the monodromy logarithm reflects the fact that we are pulling back the data of $\mathcal{V}$ to a fixed reference fiber.

More specifically, parallel translation under $\nabla$ endows our fixed reference fiber $V=\mathcal{V}_{s_{0}}$ with a choice of real structure $V_{\mathbb{R}}$ as well as a constant, real weight filtration $W$ and a collection of constant, real, non-degenerate bilinear forms

$$
\mathcal{S}_{k}: G r_{k}^{W}\left(V_{\mathbb{R}}\right) \otimes G r^{W}\left(V_{\mathbb{R}}\right) \rightarrow \mathbb{R}
$$

of alternating parity $(-1)^{k}$. Modulo the action of the monodromy group $\Gamma$, we also obtain a Hodge filtration $F_{s}$, which pairs with $W$ to define a graded-polarized mixed Hodge structure with constant graded Hodge numbers

$$
h^{p, q}=\operatorname{dim} H_{s}^{p, q}
$$

Consider the corresponding classifying space

$$
\mathcal{M}=\mathcal{M}\left(W, \mathcal{S}, h^{p, q}\right),
$$

consisting of all filtrations $F$ of $V$ such that $(F, W)$ is a mixed Hodge structure, which is graded-polarized by $\mathcal{S}$ and such that

$$
\operatorname{dim}_{\mathbb{C}} F^{p} G r_{k}^{W}=\sum_{r \geq p} h^{r, k-r}
$$


As shown in [14] and [17], $\mathcal{M}$ is a homogeneous complex manifold which fits into an ascending sequence of homogeneous spaces

$$
M_{\mathbb{R}} \subseteq \mathcal{M} \subseteq \check{\mathcal{M}} \subseteq \check{\mathcal{F}}(W) \subseteq \check{\mathcal{F}}
$$

defined as follows:

- $\check{\mathcal{F}}$ is the flag variety consisting of all decreasing filtrations $F$ such that $\operatorname{dim}_{\mathbb{C}} F^{p}=\sum_{r \geq p} h^{r, s}$.

- $\check{\mathcal{F}}(W)$ is the submanifold of $\check{\mathcal{F}}$ consisting of those filtrations $F \in \check{\mathcal{F}}$ such that $\operatorname{dim}_{\mathbb{C}} F^{p} G r_{k}^{W}=\sum_{r \geq p} h^{r, k-r}$.

- $\check{\mathcal{M}}$ is the submanifold of $\check{\mathcal{F}}(W)$ consisting of all filtrations $F \in \check{\mathcal{F}}(W)$ which satisfy Riemann's first bilinear relation with respect to the graded-polarizations $\mathcal{S}$.

- $\mathcal{M}_{\mathbb{R}}$ is the $C^{\infty}$-submanifold of $\mathcal{M}$ consisting of the filtration $F \in \mathcal{M}$ for which the associated mixed Hodge structure $(F, W)$ is split over $\mathbb{R}$.

The corresponding sequence of Lie groups is

$$
G_{\mathbb{R}} \subseteq G \subseteq G_{\mathbb{C}} \subseteq G L(V)^{W} \subseteq G L(V)
$$

where

- $G L(V)^{W}=\left\{g \in G L(V) \mid g: W_{k} \rightarrow W_{k} \quad \forall k\right\}$.

- $G_{\mathbb{C}}=\left\{g \in G L(V)^{W} \mid G r(g) \in A u t_{\mathbb{C}}(\mathcal{S})\right\}$.

$-G=\left\{g \in G_{\mathbb{C}} \mid G r(g) \in \operatorname{Aut}_{\mathbb{R}}(\mathcal{S})\right\}$.

$-G_{\mathbb{R}}=\left\{g \in G \mid g \in G L\left(V_{\mathbb{R}}\right)\right\}$.

i.e. $G L(V)$ acts transitively on $\check{\mathcal{F}}, G L(V)^{W}$ acts transitively on $\check{\mathcal{F}}(W), G_{\mathbb{C}}$ acts transitively on $\check{\mathcal{M}}, G$ acts transitively on $\mathcal{M}$ and $G_{\mathbb{R}}$ acts transitively on $\mathcal{M}_{\mathbb{R}}$.

Next we describe the canonical extension of $\mathcal{V} \rightarrow \Delta^{*}$ in more detail [11]. Given a flat vector bundle $E \rightarrow \Delta^{* n}$ with unipotent monodromy, there exists a unique extension $E^{c} \rightarrow \Delta^{n}$ relative to which the flat connection of $E$ has at worst simple poles with nilpotent residues along the divisor $D=\Delta^{n} / \Delta^{* n}$. Alternatively, given a choice of local coordinates $\left(s_{1}, \ldots, s_{n}\right)$ on $\Delta^{n}$ relative to which the divisor $D$ assumes the form $s_{1} \cdots s_{n}=0$, the canonical extension $E^{c}$ described above may be identified with the locally free sheaf generated by the sections

$$
\tilde{\sigma}=\exp \left(\frac{1}{2 \pi i} \sum_{j=1}^{n} \log s_{j} N_{j}\right) \sigma
$$

where $\sigma$ is a flat, multivalued section of $E$ and

$$
N_{j}=-\log \left(T_{j}\right)
$$

is the monodromy logarithm associated to pulling back along the $j$ 'th loop

$$
\gamma_{j}(t)=\left(s_{1}, \ldots, e^{2 \pi i t} s_{j}, \ldots, s_{n}\right) .
$$

REMARK. We shall denote the adjoint action of $G L(V)$ upon $E n d(V)$ by $g . \alpha$ and the action of $G_{\mathbb{C}}$ [resp. $G$ ] upon $\check{\mathcal{M}}$ [resp. $\mathcal{M}$ ] by $g . F$.

LEMMA 2.1. Let $\mathcal{V} \rightarrow \Delta^{*}$ be a variation of graded-polarized mixed Hodge structure with unipotent monodromy action $T=e^{-N}$, and $F: U \rightarrow \mathcal{M}$ be a lift of the associated 
period map $\varphi: \Delta^{*} \rightarrow \mathcal{M} / \Gamma$ to the upper half-plane. Then, the functions

$$
\psi(z)=e^{-z N} \cdot F(z), \quad Y(z)=e^{-z N} \cdot Y_{(F(z), W)}
$$

satisfy the periodicity conditions

$$
\psi(z+1)=\psi(z), \quad Y(z+1)=Y(z)
$$

and hence descend to well defined functions $\psi(s)$ and $Y(s)$ on the punctured disk $\Delta^{*}$ via the covering map $z \rightarrow s=e^{2 \pi i z}$. Moreover,

- The Hodge filtration $\mathcal{F}$ of $\mathcal{V}$ extends to the canonical extension $\tilde{\mathcal{V}} \rightarrow \Delta$ if and only if the limiting Hodge filtration

$$
F_{\infty}=\lim _{s \rightarrow 0} \psi(s)
$$

exists as an element of $\check{\mathcal{M}}$.

- The grading $\mathcal{Y}$ of the underlying weight filtration $\mathcal{W}$ defined by the $I^{p, q}$ 's of $\mathcal{V}$ extends continuously to the canonical extension of $\mathcal{V}$ as a grading of $\mathcal{W}$ if and only if

$$
Y_{\infty}=\lim _{s \rightarrow 0} Y(s)
$$

exists, and grades $\mathcal{W}$.

Proof. One simply identifies $\tilde{\mathcal{V}}$ with the locally free sheaf generated by the sections $\tilde{\sigma}$ described above.

For a coordinate-free description, both the limiting Hodge filtration $F_{\infty}$ and the limiting grading $Y_{\infty}$ defined above should actually be viewed as objects defined on the cotangent space of $\Delta$ at zero. More precisely, if $\tilde{s}$ and $s$ are holomorphic coordinates on $\Delta$ which vanish at 0 , then corresponding limiting objects $F_{\infty}$ and $Y_{\infty}$ defined above will in general agree if and only if $(d \tilde{s})_{0}=(d s)_{0}$.

Recall that a variation of graded-polarized mixed Hodge structure with unipotent monodromy action $T=e^{-N}$ is admissible [23] if and only if the following hold:

(1) The Hodge filtration $\mathcal{F}$ of $\mathcal{V} \rightarrow \Delta^{*}$ extends holomorphically to the canonical extension $\tilde{\mathcal{V}}$.

(2) The relative weight filtration ${ }^{r} W={ }^{r} W(N, W)$ exists.

The relative weight filtration is defined by the Lemma below. In the appendix to [23], Deligne proved that whenever a variation of graded-polarized mixed Hodge structure is admissible, then the limiting Hodge filtration $F_{\infty}$ of $\mathcal{V}$ pairs with the corresponding relative weight filtration ${ }^{r} W$ to define a mixed Hodge structure for which $N$ is a $(-1,-1)$-morphism.

To define the filtration ${ }^{r} W={ }^{r} W(N, W)$, recall first that given a nilpotent endomorphism $N$ of a finite dimensional complex vector space $V$, the corresponding (monodromy) weight filtration $W(N)$ is the unique increasing filtration of $V$ such that, for all $\ell$ :

$-N: W_{\ell}(N) \rightarrow W_{\ell-2}(N)$.

- The induced map $N^{\ell}: G r_{\ell}^{W(N)} \rightarrow G r_{-\ell}^{W(N)}$ is an isomorphism. 
Given an increasing filtration $W$ of a finite dimensional vector space $V$ and an index $\ell \in \mathbb{Z}$, the corresponding shifted object is the filtration

$$
W[\ell]_{j}=W_{j+\ell}
$$

Lemma 2.2. [23] Let $W$ be an increasing filtration of a finite dimensional vector space $V$ and $N$ be a nilpotent endomorphism of $V$ which preserves $W$. Then, there exists at most one increasing filtration ${ }^{r} W={ }^{r} W(N, W)$ of $V$ such that for each index $k$ :

(i) $N:{ }^{r} W_{k} \rightarrow{ }^{r} W_{k-2}$.

(ii) ${ }^{r} W$ induces on $G r_{k}^{W}$ the shifted monodromy weight filtration

$$
W\left(N: G r_{k}^{W} \rightarrow G r_{k}^{W}\right)[-k]
$$

of $N$ on $\mathrm{Gr}^{W}$.

To continue, we now recall the following analytic version of the nilpotent orbit theorem [17]:

LEMMA 2.3. Let $F(z)$ be the period map of a variation of graded-polarized mixed Hodge structure with unipotent monodromy for which the limiting Hodge filtration $F_{\infty}=\lim _{I m(z) \rightarrow \infty} e^{-z N} . F(z)$ exists. Then, given a choice of a vector space decomposition

$$
\operatorname{Lie}\left(G_{\mathbb{C}}\right)=\operatorname{Lie}\left(G_{\mathbb{C}}^{F_{\infty}}\right) \oplus q
$$

there exists a unique holomorphic function $\Gamma: \Delta \rightarrow q, \Gamma(0)=0, s=e^{2 \pi i z}$, such that

$$
F(z)=e^{z N} e^{\Gamma(s)} \cdot F_{\infty}
$$

for $\operatorname{Im}(z)>>0$.

Proof. One simply notes that the map $e^{-z N} \cdot F(z)$ takes values in the homogeneous space $\check{\mathcal{M}}$, upon which $G_{\mathbb{C}}$ acts transitively. To obtain $\Gamma(0)=0$, note the definition of $F_{\infty}$

REMARK. If $F(z)$ is unipotent in the sense of [13], i.e. the induced variations on $G r^{W}$ are constant, the function $\Gamma(s)$ of (2.3) assumes values in the in the subalgebra

$$
\operatorname{Lie}_{-1}=\left\{\alpha \in \operatorname{End}(V) \mid \alpha: W_{k} \rightarrow W_{k-1}\right\} \subseteq \operatorname{Lie}\left(G_{\mathbb{C}}\right)
$$

A structure of graded-polarized mixed Hodge structure $(F, W)$ on $V_{\mathbb{R}} \otimes \mathbb{C}$ induces one on the Lie algebra $\operatorname{Lie}\left(G_{\mathbb{C}}\right)$. Moreover, if $V=\bigoplus_{p, q} I^{p, q}$ is the bigrading of $V$ defined by $(F, W)$, then the corresponding decomposition of $\operatorname{Lie}\left(G_{\mathbb{C}}\right)$ is given by the subspaces

$$
g l(V)^{r, s}=\left\{\alpha \in \operatorname{Lie}\left(G_{\mathbb{C}}\right) \mid \alpha: I^{p, q} \rightarrow I^{r, s}\right\}
$$

Accordingly, $\operatorname{Lie}\left(G_{\mathbb{C}}\right)=\operatorname{Lie}\left(G_{\mathbb{C}}^{F}\right) \oplus q$, where

$$
\operatorname{Lie}\left(G_{\mathbb{C}}^{F}\right)=\eta_{+} \oplus \eta_{0}, \quad q=\eta_{-} \oplus \Lambda^{-1,-1}
$$

and

$$
\begin{array}{ll}
\eta_{+}=\bigoplus_{r \geq 0, s<0} g l(V)^{r, s}, & \eta_{-}=\bigoplus_{s \geq 0, r<0} g l(V)^{r, s} \\
\eta_{0}=g l(V)^{0,0}, & \Lambda^{-1,-1}=\bigoplus_{r, s<0} g l(V)^{r, s} .
\end{array}
$$


The decomposition

$$
\operatorname{Lie}\left(G_{\mathbb{C}}\right)=\eta_{+} \oplus \eta_{0} \oplus \eta_{-} \oplus \Lambda^{-1,-1}
$$

determines projection operators

$$
\begin{aligned}
\pi_{+}: \operatorname{Lie}\left(G_{\mathbb{C}}\right) \rightarrow \eta_{+}, & \pi_{-}: \operatorname{Lie}\left(G_{\mathbb{C}}\right) \rightarrow \eta_{-}, \\
\pi_{0}: \operatorname{Lie}\left(G_{\mathbb{C}}\right) \rightarrow \eta_{0}, & \pi_{\Lambda}: \operatorname{Lie}\left(G_{\mathbb{C}}\right) \rightarrow \Lambda^{-1,-1},
\end{aligned}
$$

The congruence (1.1) is reflected in the following relations

$$
\begin{array}{ll}
\bar{\eta}_{+} \subseteq \eta_{-} \oplus \Lambda^{-1,-1}, & \bar{\eta}_{0} \subseteq \eta_{0} \oplus \Lambda^{-1,-1}, \\
\bar{\eta}_{-} \subseteq \eta_{+} \oplus \Lambda^{-1,-1}, & \overline{\Lambda^{-1,-1}}=\Lambda^{-1,-1} .
\end{array}
$$

Note that the subalgebra $\Lambda^{-1,-1}$ depends only upon the mixed Hodge structure $(F, W)$ and not the choice of graded-polarization.

Lemma 2.7 [14][17]. Let $(F, W)$ be a mixed Hodge structure. Then,

$$
g \in \exp \left(\Lambda_{(F, W)}^{-1,-1}\right) \Longrightarrow I_{(g . F, W)}^{p, q}=g \cdot I_{(F, W)}^{p, q}
$$

In particular, $F \in \mathcal{M}$ and $g \in G_{\mathbb{R}} \cup \exp \left(\Lambda_{(F, W)}^{-1,-1}\right)$ implies $I_{(g . F, W)}^{p, q}=g \cdot I_{(F, W)}^{p, q}$.

Given $F \in \mathcal{M}$, we can smoothly decompose [14][17]

$$
g_{\mathbb{C}}=g_{\mathbb{R}} e^{\lambda} f
$$

for $g_{\mathbb{C}} \in G_{\mathbb{C}}, g_{\mathbb{C}} \approx 1$, with

$$
g_{\mathbb{R}} \in G_{\mathbb{R}}, \quad e^{\lambda} \in \exp \left(\Lambda^{-1,-1}\right), \quad f \in G_{\mathbb{C}}^{F}
$$

Similarly,

$$
g=g_{\mathbb{R}} e^{\lambda} f
$$

for $g \in G$, with

$$
g_{\mathbb{R}} \in G_{\mathbb{R}}, \quad e^{\lambda} \in \exp \left(\Lambda^{-1,-1}\right), \quad f \in G_{-1}^{F}=G_{\mathbb{C}}^{F} \cap \exp \left(\text { Lie }_{-1}\right)
$$

Therefore (2.8) holds whenever $g_{\mathbb{C}} \cdot F \in \mathcal{M}$.

For $g \in \exp \left(L_{i e_{-1}}\right)$ we can be more precise. Define an extended Lie monomial to be a non-zero function $\mu: L_{i e_{-1}} \rightarrow L i e_{-1}$ which can be obtained from the primitive monomial $m(\alpha)=\alpha$ in a finite number of steps via the operations of complex conjugation, taking Hodge components with respect to $(F, W)$ and forming Lie brackets (e.g. $\mu(\alpha)=\left[\bar{\alpha}, \alpha^{-1,0}\right]^{-1,-1}$ ). In particular, if $\mu$ is an extended Lie monomial then so is any non-zero function of the form $\pi_{+}(\mu), \pi_{\Lambda}(\mu)$ or $\pi_{-}(\mu)$. Define

$$
L_{i e_{-r}}=\left\{\zeta \in g l(V) \mid \zeta: W_{k} \rightarrow W_{k-r}\right\}
$$

which are ideals of $\mathrm{Lie}_{-1}$.

Theorem 2.9. Let $F \in \mathcal{M}$ and $\alpha \in$ Lie $_{-1}$. Then,

$$
e^{\alpha}=e^{\gamma} e^{\lambda} e^{\phi}
$$


where $\gamma, \lambda$ and $\phi$ are extended Lie polynomials in $\alpha$ which take values in the respective subalgebras Lie $\left(G_{\mathbb{R}}\right)_{-1}=\operatorname{Lie}\left(G_{\mathbb{R}}\right) \cap \operatorname{Lie}_{-1}, \Lambda^{-1,-1}$ and $\operatorname{Lie}\left(G_{\mathbb{C}}^{F}\right)_{-1}=\operatorname{Lie}\left(G_{\mathbb{C}}^{F}\right) \cap \operatorname{Lie}_{-1}$. Moreover,

$$
\begin{aligned}
\gamma & =\pi_{-}(\alpha)+\overline{\pi_{-}(\alpha)} \bmod \text { Lie }_{-2} \\
\lambda & =\pi_{\Lambda}(\alpha)-\pi_{\Lambda}\left(\overline{\pi_{-}(\alpha)}\right) \bmod \text { Lie }_{-2} \\
\phi & =\pi_{+}(\alpha)-\pi_{+}\left(\overline{\pi_{-}(\alpha)}\right) \bmod \text { Lie }_{-2}
\end{aligned}
$$

Proof. Since Lie $_{-1}$ is nilpotent, the Campbell-Baker-Hausdorff formula for it terminates after finitely many terms. Set

$$
\gamma_{1}=\pi_{-}(\alpha)+\overline{\pi_{-}(\alpha)}, \quad \lambda_{1}=\pi_{\Lambda}(\alpha)-\pi_{\Lambda}\left(\overline{\pi_{-}(\alpha)}\right), \quad \phi_{1}=\pi_{+}(\alpha)-\pi_{+}\left(\overline{\pi_{-}(\alpha)}\right) .
$$

Because of (2.6) and $\left[\operatorname{Lie}_{-p}\right.$, Lie $\left._{-q}\right] \subseteq L i e_{-p-q}$, one has $\gamma_{1}+\lambda_{1}+\phi_{1}=\alpha$. Therefore

$$
e^{\gamma_{1}} e^{\lambda_{1}} e^{\phi_{1}}=e^{\alpha+\beta_{1}}
$$

where $\beta_{1}$ is an extended Lie polynomial with values in $L i e_{-2}$. Inductively, suppose that there exist extended Lie polynomials $\gamma_{k}$ with values in $\operatorname{Lie}\left(G_{\mathbb{R}}\right)_{-1}, \lambda_{k}$ with values in $\Lambda^{-1,-1}$ and $\phi_{k}$ with values in $\operatorname{Lie}\left(G_{\mathbb{C}}^{F}\right)_{-1}$ such that

$$
e^{\gamma_{k}} e^{\lambda_{k}} e^{\phi_{k}}=e^{\alpha+\beta_{k}}
$$

for some extended Lie polynomial $\beta_{k}$ with values in $\mathrm{Lie}_{-k-1}$. Let

$$
\begin{array}{ll}
\gamma_{k+1}=\gamma_{k}+\gamma^{\prime}, & \gamma^{\prime}=-\pi_{-}\left(\beta_{k}\right)-\overline{\pi_{-}\left(\beta_{k}\right)} \\
\lambda_{k+1}=\lambda_{k}+\lambda^{\prime}, & \lambda^{\prime}=-\pi_{\Lambda}\left(\beta_{k}\right)+\pi_{\Lambda}\left(\overline{\pi_{-}\left(\beta_{k}\right)}\right) \\
\phi_{k+1}=\phi_{k}+\phi^{\prime}, & \phi^{\prime}=-\pi_{+}\left(\beta_{k}\right)+\pi_{+}\left(\overline{\pi_{-}\left(\beta_{k}\right)}\right.
\end{array}
$$

Then $\gamma^{\prime}+\lambda^{\prime}+\phi^{\prime}=-\beta_{k}$, and hence

$$
e^{\tilde{\gamma}_{k+1}} e^{\lambda_{k+1}} e^{\phi_{k+1}}=e^{\alpha+\gamma^{\prime}+\lambda^{\prime}+\phi^{\prime}+\beta+\beta_{k+1}}=e^{\alpha+\beta_{k+1}}
$$

for some extended Lie polynomial $\beta_{k+1} \in L_{i e_{-k-2}}$. As $L i e_{-r}=0$ for some index $r=r_{0}$, this completes the proof.

To close this section, we now describe the structure of split orbits in the pure case:

DeFinition 2.11. Let $\theta(z)=e^{z N} . F$ be nilpotent orbit of pure Hodge structure. Then, $\theta(z)$ is said to be an $S L_{2}$-orbit if there exists a point $F_{o}$ in the associated classifying space $\mathcal{D}$ and a Lie homomorphism $\psi: \mathrm{S} L_{2}(\mathbb{R}) \rightarrow G_{\mathbb{R}}$ such that

$$
\theta(g \cdot \sqrt{-1})=\psi(g) \cdot F_{o}
$$

for all $g \in S L_{2}(\mathbb{R})$.

THEOREM 2.12 [20]. A nilpotent orbit of pure Hodge structure $\theta$ is an $S L_{2}$-orbit if and only if $\theta$ is spilt.

Accordingly, the classification of split orbits of pure type is equivalent to describing a certain class of representations of $S L_{2}(\mathbb{R})$. More precisely, let

$$
e=(1,0), \quad f=(0,1)
$$


denote the standard basis of $\mathbb{C}^{2}$ and $S(1)$ denote the standard representation of $s l_{2}(\mathbb{C})$ on $\mathbb{C}^{2}$ equipped with the pure Hodge structure of weight 1 obtained by declaring the vectors $e+i f$ and $e-i f$ to be of Hodge type $(1,0)$ and $(0,1)$ respectively. Equip $s l_{2}(\mathbb{C})$ with Hodge structure of weight zero induced by inclusion in $\operatorname{End}\left(\mathbb{C}^{2}, \mathbb{C}^{2}\right) \cong$ $S(1) \otimes S(1)^{*}$.

THEOREM 2.13 [20]. Let $\mathcal{D}$ be a classifying space of pure, polarized Hodge structure. Then, a pair $\left(F_{o}, \psi\right)$ consisting of a point $F_{o} \in \mathcal{D}$ and a Lie homomorphism $\psi: S L_{2}(\mathbb{R}) \rightarrow G_{\mathbb{R}}$ defines an $S L_{2}$-orbit if and only if the induced map

$$
\psi_{*}: \operatorname{sl}_{2}(\mathbb{C}) \rightarrow \operatorname{Lie}\left(G_{\mathbb{C}}\right)
$$

is a morphism of Hodge structure with respect to the standard Hodge structure on $s l_{2}(\mathbb{C})$ defined above, and the pure Hodge structure induced on Lie $\left(G_{\mathbb{C}}\right)$ by $F_{o}$.

Let $H$ be a pure Hodge structure. Then, a representation $\rho: s l_{2}(\mathbb{C}) \rightarrow \operatorname{End}(H)$ is said to be Hodge provided that $\rho$ is a morphism of Hodge structure. In particular, Theorem (2.13) asserts that $\left(F_{o}, \psi\right)$ generates an $S L_{2}$-orbit if and only if $\psi_{*}$ is Hodge with respect to $F_{o}$. The category of Hodge representations is semisimple. As such, the following theorem gives a complete description of the isomorphism classes of $S L_{2}$ orbits:

TheOREm 2.14 [20]. Let $H(m)$ denote the trivial representation of $s_{2}(\mathbb{C})$ on $\mathbb{C}$, equipped with the pure Hodge structure of type $(-m,-m)$. For $p \neq q$, let $E(p, q)$ denote the trivial representation of $s l_{2}(\mathbb{C})$ on $\mathbb{C}^{2}$ equipped with the Hodge structure of weight $p+q$ obtained by declaring the vectors $e+i f$ and $e-i f$ to be of Hodge type $(p, q)$ and $(q, p)$ respectively. Then, up to isomorphism every irreducible Hodge representation is isomorphic to one of the following two types:

(a) $H(m) \otimes S(n)$, where $S(n)=\operatorname{Sym}^{n}(S(1))$;

(b) $E(p, q) \otimes S(n)$.

3. Unipotent Variations. Let $X$ let be a smooth, complex algebraic variety. Then (cf. [4]), for each positive integer $k$, there exists a canonical admissible, variation of graded-polarized mixed Hodge structure $\mathcal{V} \rightarrow X$ with fiber

$$
\mathcal{V}_{x}=\mathbb{C} \pi_{1}(X, x) /\left(J_{x}\right)^{k+1}
$$

where $J_{x}$ is the augmentation ideal of $\mathbb{C} \pi_{1}(X, x)$. The monodromy representation

$$
\rho: \pi_{1}(X, x) \rightarrow \operatorname{Aut}\left(\mathcal{V}_{x}\right)
$$

of such a variation is unipotent and the variations of pure, polarized Hodge structure induced by $\mathcal{V}$ on $G r^{\mathcal{W}}$ are constant in this situation. Such variations are called unipotent. With this motivation in mind, we consider here the problem of determining the admissibility for such variations in terms of the grading $\mathcal{Y}$ as discussed in $\S 1$, beginning with the following observations:

(a) By virtue of Schmid's Nilpotent Orbit Theorem, the monodromy logarithm $N$ of a unipotent variation $\mathcal{V} \rightarrow \Delta^{*}$ must act trivially on $G r^{W}$.

(b) On account of $(a)$, the relative weight filtration ${ }^{r} W$ of a unipotent variation exists if and only if $N: W_{k} \rightarrow W_{k-2}$ for each index $k$, i.e. ${ }^{r} W=W$. 
THEOREM 3.1. Let $\mathcal{V} \rightarrow \Delta^{*}$ be a unipotent variation of graded-polarized mixed Hodge structure which is admissible. Then, $\mathcal{Y}$ extends continuously, as a grading of $\mathcal{W}$, to the canonical extension $\tilde{\mathcal{V}} \rightarrow \Delta$.

Proof. Let $F: U \rightarrow \mathcal{M}$ be a lift the period map of $\mathcal{V}$ to the upper half-plane $U$ and let $N$ be its monodromy logarithm. Then, as discussed in $\S 2$, the claim is equivalent to the assertion that

$$
Y_{\infty}=\lim _{\operatorname{Im}(z) \rightarrow \infty} e^{-z N} \cdot Y_{(F(z), W)}
$$

exists and grades $W$.

To verify the existence of the grading $Y_{\infty}$, observe that on account of the unipotency of $\mathcal{V}$, the function

$$
\psi(s)=e^{-z N} \cdot F(z), \quad s=e^{2 \pi i z}
$$

considered in $\S 2$ takes values in $\mathcal{M}$ (and not $\check{\mathcal{M}}$ as is a priori the case). Indeed, in our case $\psi(s)$ induces the same filtration as $F(z)$ on $G r^{W}$, and hence is an element of $\mathcal{M}$. Moreover, since the induced filtration $F(z) G r^{W}$ of a unipotent variation is by definition constant, the limiting Hodge filtration

$$
F_{\infty}=\lim _{s \rightarrow 0} \psi(s)
$$

of a $\mathcal{V}$ is likewise an element of $\mathcal{M}$. By $(b)$ above, the relative weight filtration of our admissible variation $\mathcal{V}$ must coincide with the weight filtration $W$ of $\mathcal{V}$. By Deligne's theorem, $N$ is $(-1,-1)$-morphism of $\left(F_{\infty}, W\right)$. We now apply Lemma (2.3), with $q=\eta_{-} \oplus \Lambda^{-1,-1}$ as defined by (2.4) and $F=F_{\infty}$. This gives

$$
F(z)=e^{z N} e^{\Gamma(s)} \cdot F_{\infty}, \quad \Gamma(0)=0
$$

relative to a holomorphic function $\Gamma(s)$ taking values in the subalgebra

$$
q \cap L i e_{-1}=\bigoplus_{r<0, r+s \leq-1} g l(V)^{r, s}
$$

Consequently,

$$
e^{-z N} \cdot Y_{(F(z), W)}=e^{-z N} \cdot Y_{\left(e^{z N} e^{\Gamma(s)} \cdot F_{\infty}, W\right)}
$$

To finish the proof, let $e^{X(z)}=e^{z N} e^{\Gamma(s)}$ Then

$$
e^{X(z)}=e^{z N+\Gamma(s)+(\text { brackets of } z N \text { and } \Gamma(s))}
$$

while, by Theorem (2.9),

$$
e^{X(z)}=e^{\gamma(z)} e^{\lambda(z)} e^{\phi(z)}
$$

where $\gamma(z), \lambda(z)$ and $\phi(z)$ extended Lie polynomials in $X(z)$ with respect to $\left(F_{\infty}, W\right)$. Accordingly,

$$
\begin{aligned}
e^{-z N} \cdot Y_{\left(e^{z N} e^{\Gamma(s)} \cdot F_{\infty}, W\right)} & =e^{-z N} e^{\gamma(z)} e^{\lambda(z)} \cdot Y_{\left(F_{\infty}, W\right)} \\
& =e^{-z N} e^{z N} e^{\Gamma(s)} e^{-\phi(z)} \cdot Y_{\left(F_{\infty}, W\right)} \\
& =e^{\Gamma(s)} e^{-\phi(z)} \cdot Y_{\left(F_{\infty}, W\right)}
\end{aligned}
$$

Since $\phi(z)$ is an extended Lie polynomial in $X(z)$ which takes values in $\operatorname{Lie}\left(G_{\mathbb{C}}^{F_{\infty}}\right)_{-1}$, the Hodge components $X(z)^{r, s}$ of $X(z)$ with both $r, s<0$ can only appear in $\phi(z)$ 
inside of Lie brackets which contain some Hodge component $X(z)^{p, q}$ with either $p$ or $q$ greater than zero. Indeed, since

$$
\Lambda^{-1,-1}=\bigoplus_{r, s<0} g l(V)^{r, s}
$$

is a subalgebra of $L_{i} e_{-1}$ which is closed under complex conjugation, and the extended Lie monomials are exactly the non-zero functions $\mu: L_{i e_{-1}} \rightarrow L_{i e_{-1}}$ which can by constructed in a finite number of steps from the primitive monomial $m(\alpha)=\alpha$ via the operations of complex conjugation, taking Hodge components and forming Lie

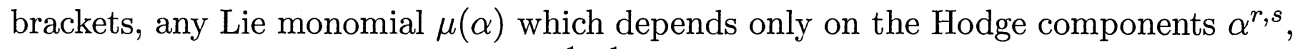
with both $r, s<0$ must preserve $\Lambda^{-1,-1}$.

Returning to (3.4) and noting that $N \in g l(V)^{-1,-1}$, it follows from the above considerations that

$$
\|\phi(z)\| \leq K|z|^{b}\left|e^{-2 \pi i z}\right|
$$

for some constants $K$ and $b$ (and $\|*\|$ a norm on Lie $_{-1}$ ). Consequently,

$$
\lim _{\operatorname{Im}(z) \rightarrow \infty} \phi(z)=0 .
$$

On account of (3.5), (3.6) and the fact that $\Gamma(0)=0$, the limit (3.2) exists and equals $Y_{\left(F_{\infty}, W\right)}$.

This finishes the proof of (3.1).

COROLlaRY 3.7. Let $\mathcal{V} \rightarrow \Delta^{*}$ be a unipotent variation of graded-polarized mixed Hodge structure which is admissible. Then, $\mathcal{Y}$ extends continuously, as a grading of $\mathcal{W}$, to the canonical prolongation of $\mathcal{V}$, with limiting value $Y_{\infty}=Y_{\left(F_{\infty}, W\right)}$.

We now establish the converse of Theorem (3.1):

THEOREM 3.8. Let $\mathcal{V} \rightarrow \Delta^{*}$ be a unipotent variation of graded-polarized mixed Hodge structure, and suppose that the grading $\mathcal{Y}$ of the underlying weight filtration $\mathcal{W}$ defined by the $I^{p, q}$ 's of $\mathcal{V}$ extends continuously, as a grading of $\mathcal{W}$, to the canonical extension $\tilde{\mathcal{V}}$. Then, $\mathcal{V}$ is admissible.

Proof. As in the proof of Theorem (3.1), we select a lifting $F: U \rightarrow \mathcal{M}$ of the period map of $\mathcal{V}$. Lemma (2.1), implies that $\mathcal{Y}$ extends continuously to a grading of $\mathcal{W}$ in the canonical extension of $V$, that

$$
Y_{\infty}=\lim _{s \rightarrow 0} Y(s), \quad Y(s)=e^{-z N} \cdot Y_{(F(z), W)}
$$

with $s=e^{2 \pi i z}$, exists and grades $W$.

To see that (3.9) implies the existence of the limiting Hodge filtration

$$
F_{\infty}=\lim _{s \rightarrow 0} \psi(s), \quad \psi(s)=e^{-z N} \cdot F(z)
$$

observe that $Y_{(F(z), W)}$ preserves the filtration $F(z)$, so $Y(s)$ preserves $\psi(s)$. Accordingly, $\psi(s)$ can be obtained by pulling back the induced filtration $\psi(s) G r^{W}$ via the isomorphism $V \cong G r^{W}$ determined by $Y(s)$, namely

$$
G r_{k}^{W} \cong E_{k}(Y(s))
$$

As the induced filtrations $\psi(s) G r^{W}$ are constant, the existence of the limiting grading (3.9) imply the existence of the limiting Hodge filtration $F_{\infty}$. 
By (b), the existence of the relative weight filtration is equivalent (in the present case) to the statement

$$
N: W_{k} \rightarrow W_{k-2}
$$

As in the proof of Theorem (3.1), the unipotency of $\mathcal{V}$ implies that $F_{\infty} \in \mathcal{M}$. We can then apply Theorem (2.3) with $F=F_{\infty}$ and $q=\eta_{-} \oplus \Lambda^{-1,-1}$. This gives

$$
F(z)=e^{z N} e^{\Gamma(s)} \cdot F_{\infty}, \quad \Gamma(s)=0
$$

relative to a holomorphic function $\Gamma(s)$ taking values in the subalgebra (3.3). Letting

$$
e^{X(z)}=e^{z N} e^{\Gamma(s)}
$$

we may write (cf. (3.5))

$$
Y(s)=e^{\Gamma(s)} e^{-\phi(z)} \cdot Y_{\left(F_{\infty}, W\right)}
$$

where $\phi(z)$ is the extended Lie polynomial in $X(z)$ obtained by decomposing $e^{X(z)}$ in accord with (2.9). Moreover, by (2.10),

$$
\phi(z)=\pi_{+}(X(z))-\pi_{+}\left(\overline{\pi_{-}(X(z))}\right) \quad \bmod \operatorname{Lie}_{-2}
$$

Claim:

$$
N: F_{\infty}^{p} \rightarrow F_{\infty}^{p-1}
$$

Indeed, by the horizontality of $F(z)$,

$$
\frac{d}{d z} F^{p}(z) \subseteq F^{p-1}(z)
$$

Inserting the formula $F(z)=e^{z N} e^{\Gamma(s)}$ in the last equality and simplifying, one then obtains

$$
e^{-\Gamma(s)} \cdot N+2 \pi i s e^{-\Gamma(s)} \frac{d}{d s} e^{-\Gamma(s)}: F_{\infty}^{p} \rightarrow F_{\infty}^{p-1}
$$

Setting $s=0,(3.11)$ follows.

Because of (3.11), the mixed-Hodge decomposition of $N$ relative to $\left(F_{\infty}, W\right)$ satisfies

$$
N=N^{0,-1}+N^{-1,0} \bmod \text { Lie }_{-2}
$$

with

$$
\overline{N^{-1,0}}=N^{0,-1} \quad \bmod \text { Lie }_{-2}
$$

In addition, by (3.4),

$$
X(z)=z N+\Gamma(s) \quad \bmod \text { Lie }_{-2}
$$

Taking note of (3.10) and (3.12)-(3.14), it then follows that

$$
\phi(z)=2 i y N^{0,-1}+\pi_{+}(\Gamma(s))-\pi_{+}\left(\overline{\pi_{-}(\Gamma(s))}\right) \quad \bmod \text { Lie }_{-2}
$$

Accordingly, the limiting grading

$$
\begin{aligned}
Y_{\infty} & =\lim _{s \rightarrow 0} Y(s)=\lim _{\operatorname{Im}(z) \rightarrow \infty} e^{-z N} \cdot Y_{(F(z), W)} \\
& =\lim _{\operatorname{Im}(z) \rightarrow \infty} e^{\Gamma(s)} e^{-\phi(z)} \cdot Y_{\infty}
\end{aligned}
$$

exists only if $N^{0,-1}=0$. Indeed, by [5], the group $\exp \left(\right.$ Lie $\left._{-1}\right)$ acts simply transitively upon the set of all gradings $Y$ of $W$. Therefore, in order for the limit (3.16) to exist, 
$\|\phi(z)\|$ must remain bounded as $\operatorname{Im}(z) \rightarrow \infty$, and hence $N^{0,-1}$ must be equal to zero by (3.16) [recall $\Gamma(0)=0$ ]. Since $N^{-1,0}=\overline{N^{0,-1}}$ by (3.13), it then follows that $N=0$ $\bmod$ Lie $_{-2}$, i.e. $N: W_{k} \rightarrow W_{k-2}$.

Combining Theorems (3.1) and (3.8), we then obtain:

THEOREM (I). Let $\mathcal{V} \rightarrow \Delta^{*}$ be a variation of graded-polarized mixed Hodge structure which is unipotent. Then, $\mathcal{V}$ is admissible if and only if the $C^{\infty}$-grading $\mathcal{Y}$ of $\mathcal{W}$ defined by the $I^{p, q}$ 's of $\mathcal{V}$ extends continuously, as a grading of $\mathcal{W}$, to the canonical extension $\tilde{\mathcal{V}}$.

4. Split Orbits. Let $\mathcal{M}=\mathcal{M}\left(W, \mathcal{S}, h^{p, q}\right)$ be a classifying space of gradedpolarized mixed Hodge structure, with "compact dual" $\check{\mathcal{M}}$ and associated Lie groups $G_{\mathbb{R}}, G$ and $G_{\mathbb{C}}$, as described in section 2 .

Definition. A nilpotent orbit of graded-polarized mixed Hodge structure (modeled on $\mathcal{M})$ consists of a filtration $F \in \check{\mathcal{M}}$ and an element $N \in \operatorname{Lie}\left(G_{\mathbb{R}}\right)$ such that

$-N: F^{p} \rightarrow F^{p-1}$ for each index $p$.

- There exists a constant $\alpha$ such that $\operatorname{Im}(z)>\alpha \Longrightarrow e^{z N} \cdot F \in \mathcal{M}$.

In this section we prove the following version of Theorem II:

THEOREM 4.1. A nilpotent orbit of graded-polarized mixed Hodge structure $e^{z N} . F$ is admissible and split if and only if

(a) The limit

$$
Y_{\infty}=\lim _{\operatorname{Im}(z) \rightarrow \infty} A d\left(e^{-z N}\right) Y_{\left(e^{z N} . F, W\right)}
$$

exists, grades $W$ and is defined over $\mathbb{R}$.

(b) Each of the induced orbits $e^{z N} . F G r_{k}^{W}$ is split

As noted in the introduction, the fact that an admissible split nilpotent orbit satisfies the conditions of the Theorem is an immediate consequence of results of [8] discussed in the attached appendix.

To prove the converse, we let

$$
\rho: s l_{2}(\mathbb{R}) \rightarrow \operatorname{End}_{\mathbb{R}}(V)
$$

denote the representation of $s l_{2}(\mathbb{R})$ constructed in $\S 1$ by simply pulling back the representations $\rho_{k}: s l_{2}(\mathbb{R}) \rightarrow E n d_{\mathbb{R}}\left(G r_{k}^{W}\right)$ to $V$ via the isomorphism $G r^{W} \cong V$ induced by $Y_{\infty}$, and define

$$
{ }^{(r)} Y=Y_{\infty}+Y_{o}, \quad Y_{o}=\rho(h)
$$

Then, as discussed in $\S 1$, in order for Theorem (4.1) to hold, ${ }^{(r)} Y$ must grade the relative weight filtration of $N$ and $W$, i.e. the associated filtration

$$
{ }^{(r)} W_{k}=\bigoplus_{\ell \leq k} E_{j}\left({ }^{(r)} Y\right)
$$

must satisfy the following two conditions (cf. (2.2)):

(i) $N:{ }^{(r)} W_{k} \rightarrow{ }^{(r)} W_{k-2}$.

(ii) ${ }^{(r)} W$ induces on $G r_{k}^{W}$ the shifted monodromy weight filtration

$$
W^{\#}(k)=W\left(N: G r_{k}^{W} \rightarrow G r^{W}\right)[-k]
$$

of $N$ on $G r^{W}$. 
To facilitate the proof we record the following observations:

(1) The limiting grading $Y_{\infty}$ from (a) in Theorem (4.1), preserves $F$.

(2) $Y_{o}=\rho(h)$ preserves $F$.

(3) Suppose that ${ }^{(r)} Y$ does indeed grade the relative weight filtration of $N$ and $W$. Then $\left(F,{ }^{r} W\right)$ is split over $\mathbb{R}$. In particular, the proof of Theorem (4.1) will be complete once we verify (i) and (ii) above.

(4) The representation $\rho$ defined above acts on $G r^{W}$ by infinitesimal isometries (i.e. $\rho$ takes values in $\operatorname{Lie}\left(G_{\mathbb{R}}\right)$.

To prove (1), observe that $e^{-z N} \cdot Y_{\left(e^{z N} . F, W\right)}$ preserves $F$. To prove (2), we recall from [20] that:

$$
\rho_{k}(h)=Y_{\left(F G r_{k}^{W}, W \#(k)\right)}-k
$$

To prove (3), note that items (1) and (2) together with the hypothesis of Theorem, imply that ${ }^{(r)} Y=Y_{\infty}+Y_{o}$ is a semisimple endomorphism of $V$ which preserves $F$. Accordingly, if ${ }^{(r)} Y$ also grades ${ }^{r} W$ then $\left(F,{ }^{r} W\right)$ must be a split mixed Hodge structure. The proof of (4) can be found in [18] and boils down to the assertion that the monodromy weight filtration $W\left(N: G r_{k}^{W} \rightarrow G r_{k}^{W}\right)$ is self-dual with respect to the polarization $\S_{k}$.

To verify (ii), one simply observes that on account of the formula for $\rho_{k}$ given above, ${ }^{(r)} Y=Y_{\infty}+\rho(h)$ acts on $G r_{k}^{W}$ as $Y_{(F, W \#(k))}$.

To establish $(i)$, we shall actually verify a (seemingly) stronger condition, namely

$$
\left[{ }^{(r)} Y, N\right]=-2 N
$$

To this end, we let

$$
N=N_{0}+N_{-1}+\cdots
$$

denote the decomposition of $N$ with respect to the eigenvalues of $a d Y_{\infty}$ and recall that in the present context

$$
Y_{o}=\rho(h), \quad N_{0}=\rho\left(n_{o}\right)
$$

with

$$
n_{o}=\left(\begin{array}{cc}
0 & 0 \\
1 & 0
\end{array}\right), \quad h=\left(\begin{array}{cc}
1 & 0 \\
0 & -1
\end{array}\right), \quad n_{o}^{+}=\left(\begin{array}{ll}
0 & 1 \\
0 & 0
\end{array}\right)
$$

Accordingly, $\left[{ }^{(r)} Y, N\right]=-2 N$ if and only if

$$
\left[Y_{o}, N_{-k}\right]=(k-2) N_{-k}
$$

for each index $k>0$. Consequently, it will suffice to prove the following assertion:

LEMMA 4.2. Relative to the representation $\rho$ of $\operatorname{sl}_{2}(\mathbb{R})$ defined above, any nonzero component $N_{-k}$ with $k>0$ is a highest weight vector of weight exactly $(k-2)$.

For the remainder of this section, we shall write $Y_{F}$ in place of $Y_{(F, W)}$ whenever $F \in \mathcal{M}$.

By virtue of (a), we know that:

$$
Y_{\infty}=\lim _{y \rightarrow \infty} e^{-i y N} \cdot Y_{e^{i y N} . F}
$$


Furthermore, we also know that $N$ is horizontal with respect to $F$, and hence

$$
N_{-k}: F^{p} \rightarrow F^{p-1}
$$

since $Y_{\infty}$ preserves $F$. Accordingly, we can prove Lemma (4.2) inductively by explicitly computing the right hand side of (4.3) and then imposing the horizontality condition (4.4).

To fill in the details, observe that Schmid's $S L_{2}$ Orbit Theorem applied to $G r^{W}$ (a sum of variations of pure structures) together with (b), implies that the pair

$$
\left(e^{i y N_{0}} \cdot F, W\right)
$$

is a mixed Hodge structure for all $y>0$. In particular, the base point

$$
F_{o}=e^{i N_{0}} \cdot F
$$

is an element of $\mathcal{M}$. Moreover, since $Y_{\infty}$ is both real and preserves the filtration $e^{i y N_{0}} . F$,

$$
Y_{\infty}=Y_{\left(e^{i y N_{0}}, F, W\right)}
$$

for all $y>0$.

Next, we introduce the $\exp \left(\mathrm{Lie}_{-1}\right)$-valued function

$$
e^{Q(y)}=e^{i y N} e^{-i y N_{0}}=e^{i y N_{0}+i y N^{\prime}} e^{-i y N_{0}}, \quad N^{\prime}=N-N_{0}
$$

and note that

$$
e^{i y N}=e^{Q(y)} e^{i y N_{0}}=y^{-\frac{1}{2} Y_{o}} e^{P(y)} e^{i N_{0}} y^{\frac{1}{2} Y_{o}}
$$

upon setting $P(y)=A d\left(y^{\frac{1}{2} Y_{o}}\right) Q(y)$. In particular, since $y^{\frac{1}{2} Y_{o}}$ preserves $F$ :

$$
e^{i y N} \cdot F=y^{-\frac{1}{2} Y_{o}} e^{P(y)} e^{i N_{0}} y^{\frac{1}{2} Y_{o}} \cdot F=y^{-\frac{1}{2} Y_{o}} e^{P(y)} \cdot F_{o}
$$

Thus, upon applying Theorem (2.9) to $e^{P(y)}$ with $F=F_{o}$, we obtain a distinguished decomposition

$$
e^{P(y)}=e^{\gamma(y)} e^{\lambda(y)} f(y), \quad f(y)=e^{\phi(y)}
$$

such that

$$
I_{\left(e^{P(y)} \cdot F_{o}, W\right)}^{p, q}=e^{P(y)} f^{-1}(y) \cdot I_{\left(F_{o}, W\right)}^{p, q}
$$

Consequently,

$$
\begin{aligned}
Y_{e^{i y N} . F} & =y^{-\frac{1}{2} Y_{o}} \cdot Y_{e^{P(y)} \cdot F_{o}}=y^{-\frac{1}{2} Y_{o}} e^{P(y)} f^{-1}(y) \cdot Y_{\left(F_{o}, W\right)} \\
& =y^{-\frac{1}{2} Y_{o}} e^{P(y)} f^{-1}(y) \cdot Y_{\infty}
\end{aligned}
$$

where from [cf. (4.7)]

$$
\begin{aligned}
e^{-i y N} . Y_{e^{i y N} . F} & =e^{-i y N} y^{-\frac{1}{2} Y_{o}} e^{P(y)} f^{-1}(y) \cdot Y_{\infty} \\
& =\left(y^{-\frac{1}{2} Y_{o}} e^{P(y)} e^{i N_{0}} y^{\frac{1}{2} Y_{o}}\right)^{-1} y^{-\frac{1}{2} Y_{o}} e^{P(y)} f^{-1}(y) . Y_{\infty} \\
& =y^{-\frac{1}{2} Y_{o}} e^{-i N_{0}} f^{-1} \cdot Y_{\infty} \\
& =y^{-\frac{1}{2} Y_{o}} e^{-i N_{0}} f^{-1}(y) e^{i N_{0}} \cdot Y_{\infty}
\end{aligned}
$$

with the very last step being justified by the fact that $e^{i N_{0}} \cdot Y_{\infty}=Y_{\infty}$. 
Since $\left[Y_{o}, Y_{\infty}\right]=0$

$$
e^{-i y N} \cdot Y_{e^{i y N} . F}=\left[A d\left(y^{-\frac{1}{2} Y_{o}}\right) A d\left(e^{-i N_{o}}\right) f^{-1}(y)\right] . Y_{\infty}
$$

In particular, since $f^{-1}(y)$ takes values in $\exp \left(\right.$ Lie $\left._{-1}\right),(4.3)$ holds if and only if

$$
\lim _{y \rightarrow \infty} A d\left(y^{-\frac{1}{2} Y_{o}}\right) A d\left(e^{-i N_{o}}\right) f^{-1}(y)=1
$$

Equivalently, if $f(y)=e^{B(y)}$, then

$$
\lim _{y \rightarrow \infty} A d\left(y^{-\frac{1}{2} Y_{o}}\right) A d\left(e^{-i N_{o}}\right) B(y)=0
$$

Write

$$
B(y)=\sum B_{m} y^{\frac{m}{2}}
$$

Then,

$$
A d\left(y^{-\frac{1}{2} Y_{o}}\right) A d\left(e^{-i N_{0}}\right) B(y)=\sum_{m} \sum_{j}\left[A d\left(e^{-i N_{0}}\right) B_{m}\right]_{j}^{Y_{o}} y^{\frac{m-j}{2}}
$$

and hence (4.7) holds if and only if

$$
\left[e^{-i a d N_{0}} B_{m}\right]_{j}^{Y_{o}}=0, \quad \forall j \leq m
$$

Let $[A]_{\lambda}^{S}$ denote the component of $A$ in the $\lambda$-eigenspace of a semisimple element $S \in \operatorname{End}(V)$.

To compute $B(y)$, note that (cf. Appendix):

$$
\begin{aligned}
e^{Q(y)} & =e^{\left(i y N_{0}+i y N^{\prime}\right)} e^{-i y N_{0}} \\
& =\exp \left(\Psi\left(i y N^{\prime}, \ldots, \frac{1}{m !} \operatorname{ad}\left(i y N_{0}\right)^{m}\left(i y N^{\prime}\right), \ldots\right)\right)
\end{aligned}
$$

for some Lie polynomial $\Psi$ such that

$$
Q(y)=\frac{e^{a d\left(i y N_{0}\right)}-1}{a d\left(i y N_{0}\right)}\left(i y N^{\prime}\right)+O\left(\left(i y N^{\prime}\right)^{2}\right)
$$

where $O\left(\left(i y N^{\prime}\right)^{2}\right)$ is of "lower weight", i.e. if $N^{\prime}=N_{-k}$, this is a decomposition of $Q(y)$ according to $E_{-k}\left(Y_{\infty}\right)+L i e_{-2 k}$. Consequently,

$$
P(y)=\frac{e^{a d\left(i N_{0}\right)}-1}{a d\left(i N_{0}\right)}\left(i N^{\prime}(y)\right)+U_{[-2]}(y)
$$

where

$$
N^{\prime}(y)=y A d\left(y^{\frac{Y_{o}}{2}}\right) N^{\prime}=\sum_{k \geq 1} \sum_{j}\left[N_{-k}\right]_{j}^{Y_{o}} y^{1+\frac{1}{2} j}
$$

and $U_{[-2]}=y^{2} A d\left(y^{\frac{Y_{o}}{2}}\right) O\left(\left(N^{\prime}\right)^{2}\right)$ is of lower weight.

Now, since $\rho$ induces a Hodge representations on $G r^{W}$ with respect to $F_{o} G r^{W}$ and $Y_{\infty}$ is real, commutes with $\rho$ and preserves $F_{o}, \operatorname{Lie}\left(G_{\mathbb{C}}\right)$ can be decomposed into a direct sum of irreducible Hodge modules, each of which is an eigenspace of $a d Y_{\infty}$. By Theorem (2.14), up to isomorphism, such a module is either of the form $H(m) \otimes S(n)$ 
or $E(p, q) \otimes S(n)$. This fact has the following consequence: Let $\left(x^{-}, z, x^{+}\right)$denote the $s l_{2}$-triple defined by the matrices

$$
\begin{gathered}
x^{-}=\frac{1}{2}\left(h-i n_{o}-i n_{o}^{+}\right) \quad x^{+}=\frac{1}{2}\left(h+i n_{o}+i n_{o}^{+}\right) \\
z=i\left(n_{o}-n_{o}^{+}\right)
\end{gathered}
$$

where $\left(n_{o}, h, n_{o}^{+}\right)$are as on page 323 . Then, relative to the standard Hodge structure on $s l_{2}(\mathbb{C})$ defined at the end of section $2, x^{-}, z$ and $x^{+}$are of Hodge type $(-1,1),(0,0)$, $(1,-1)$. Accordingly, if $Z=\rho(z)$ and $U \subseteq E_{-k}^{Y_{\infty}}$ is an irreducible Hodge representation of $\operatorname{Lie}\left(G_{\mathbb{C}}\right)$ of type $H(m) \otimes S(n)$ then

$$
E_{k+2 r}^{Z}(U)=U^{-r,-k+r}
$$

REMARK 4.13. When $U$ is of type $E(p, q) \otimes S(n)$, equation (4.12) must be corrected as follows: Let $U=U^{\prime} \oplus U^{\prime \prime}$ denote the decomposition of $U \cong E(p, q) \otimes S(n)$ into irreducible $s l_{2}$-modules defined by setting $U^{\prime}=\operatorname{span}(e+i f) \otimes S(n)$ and $U^{\prime \prime}=$ $\operatorname{span}(e-i f) \otimes S(n)$. Then, on $U^{\prime}, k$ get replaced by $k-\delta$ where $\delta=p-q$. Likewise, on $U^{\prime \prime}, k$ is replaced by $k+\delta$.

We may now proceed with the proof of Lemma (4.2). Inductively, we may assume that it holds for eigenvalues of $a d Y_{\infty}$ bigger than $-k$. In particular, since the basis $\left(X^{-}, Z, X^{+}\right)$given by

$$
X^{-}=\rho\left(x^{-}\right), \quad Z=\rho(z), \quad X^{+}=\rho\left(x^{+}\right)
$$

defines the same representation of $s l_{2}(\mathbb{C})$ as $\left(N_{0}, Y_{o}, N_{0}^{+}\right)$, the monomials

$$
\left(a d N_{0}\right)^{j} N_{-\ell}, \quad-\ell>-k, \quad 0 \leq j \leq \ell-2
$$

belong to $\Lambda_{\left(F_{0}, W\right)}^{-1,-1}$ on account of (4.12). Consequently, if we decompose

$$
P(y)=P_{-1}(y)+P_{-2}(y)+\cdots
$$

according to the eigenvalues of $a d Y_{\infty},(4.14)$ and (4.9) imply that

$$
P(y)=P_{\mathbb{R}}(y)+P_{\Lambda}(y)+B(y) \quad \bmod L i e_{-k-1}
$$

with $P_{\mathbb{R}}(y) \in \operatorname{Lie}\left(G_{\mathbb{R}}\right)_{-1}, P_{\Lambda}(y) \in \Lambda_{\left(F_{o}, W\right)}^{-1,-1}$ and

$$
P_{-k}(y)=\frac{e^{a d\left(i N_{0}\right)}-1}{a d\left(i N_{0}\right)}\left(i \sum_{m} y^{1+\frac{1}{2} m}\left[N_{-k}\right]_{m}^{Y_{o}}\right) \quad \bmod \Lambda_{\left(F_{o}, W\right)}^{-1,-1}
$$

Indeed: (4.9) gives a formula for $P(y)$ in terms of the monomials $\left(a d N_{0}\right)^{r} N_{-s}$ and their projections. By inductive hypothesis and the fact that $\Lambda_{\left(F_{o}, W\right)}^{-1,-1}$ is an associative subalgebra of $g l(V)$, the monomials listed in (4.14) can only contribute factors which belong to $\Lambda_{\left(F_{o}, W\right)}^{-1,-1}$.

Together with (4.12), this shows

$$
B(y)=\operatorname{proj}_{F_{o}}\left(\frac{e^{a d\left(i N_{0}\right)}-1}{a d\left(i N_{0}\right)} \sum_{m} i y^{1+\frac{1}{2} m}\left[N_{-k}\right]_{m}^{Y_{o}}\right) \quad \bmod \text { Lie }_{-k-1}
$$


where, in general, we shall let

$$
\operatorname{proj}_{F_{o}}: \operatorname{Lie}\left(G_{\mathbb{C}}\right) \rightarrow \operatorname{Lie}\left(G_{\mathbb{C}}^{F_{o}}\right)
$$

denote the projection operator defined by (4.9) via the rule:

$$
\operatorname{proj}_{F_{o}}\left(\sum_{r, s} \alpha^{r, s}\right)=\sum_{r \geq 0, s} \alpha^{r, s}
$$

Accordingly, by (4.17), the coefficients of the series expansion $B(y)=\sum_{m} B_{m} y^{\frac{1}{2} m}$ are given by the formula

$$
B_{m}=\operatorname{proj}_{F_{o}}\left(\frac{e^{a d\left(i N_{0}\right)}-1}{a d\left(N_{0}\right)}\left[N_{-k}\right]_{m-2}^{Y_{o}}\right) \bmod L i e_{-1-k}
$$

Returning now to the setting of (4.12), let us restrict our attention to an irreducible Hodge module

$$
U \subseteq E_{-k}\left(a d Y_{\infty}\right)
$$

of highest weight $d$ and type $H(m) \otimes S(n)$. Then, by virtue of our preceding remarks, we obtain the identity

$$
B_{m}=\operatorname{proj}_{F_{o}}\left(\frac{e^{a d\left(i N_{0}\right)}-1}{a d\left(N_{0}\right)}\left[N_{-k}\right]_{m-2}^{Y_{o}}\right)
$$

To continue, we note that for positive values of $k$, we may compute $\operatorname{proj}_{F_{o}}(T)$ for any element $T \in E_{-k}\left(Y_{\infty}\right)$ via the formula

$$
\operatorname{Lie}\left(G_{\mathbb{C}}\right)=\operatorname{Lie}\left(G_{\mathbb{R}}\right)+\Lambda_{\left(F_{o}, W\right)}^{-1,-1}+\operatorname{Lie}\left(G_{\mathbb{C}}^{F_{o}}\right)
$$

by determining its Hodge components: replacing $T$ by $\frac{1}{2}(T-\bar{T})$ gets rid of the real part, and taking just the components of type $\ldots,(1,-k-1),(0,-k)$ removes the contribution from $\Lambda^{-1,-1}$. Thus,

$$
\operatorname{proj}_{F_{o}}(T)=\frac{1}{2} \sum_{a \geq 0}(T-\bar{T})^{a,-k-a}
$$

In particular,

$$
\begin{aligned}
B_{m} & =\operatorname{proj}_{F_{o}}\left(\frac{e^{a d\left(i N_{0}\right)}-1}{a d\left(N_{0}\right)}\left[N_{-k}\right]_{m-2}^{Y_{o}}\right) \\
& =\frac{1}{2} \sum_{a \geq 0}\left(\left(\frac{e^{a d\left(i N_{0}\right)}-1}{a d\left(N_{0}\right)}-\frac{e^{-a d\left(i N_{0}\right)}-1}{a d\left(N_{0}\right)}\right)\left[N_{-k}\right]_{m-2}^{Y_{o}}\right)^{a,-k-a} \\
& =i \sum_{a \geq 0}\left(\frac{\sin a d\left(N_{0}\right)}{a d\left(N_{0}\right)}\left[N_{-k}\right]_{m-2}^{Y_{o}}\right)^{a,-k-a}
\end{aligned}
$$

Therefore, by (4.12):

$$
B_{m}=i \sum_{\ell \geq k}\left[\frac{\sin a d\left(N_{0}\right)}{a d\left(N_{0}\right)}\left[N_{-k}\right]_{m-2}^{Y_{o}}\right]_{\ell}^{Z}
$$


Since $F_{o}=e^{i N_{0}} . F$, the horizontality of $N_{-k}$ at $F$ together with (4.12) imply that

$$
e^{i a d N_{0}} N_{-k} \in \bigoplus_{a \geq-1} g l(V)_{\left(F_{o}, W\right)}^{a,-k-a}=\bigoplus_{\ell \geq k-2} E_{\ell}^{Z}
$$

and hence

$$
d \geq k-2 \text {. }
$$

(4.19) is equivalent to the assertion that

$$
N_{-k} \in \bigoplus_{\ell \geq k-2} E_{\ell}^{Y_{o}}
$$

since $e^{i a d N_{0}}$ is actually an isomorphism from $E_{\ell}^{Y_{o}}$ to $E_{\ell}^{Z}$ (see proof of Lemma (4.23) below).

Corollary. By virtue of horizontality, $B_{m}=0$ for $m<k$.

Proof. Indeed, by (4.20), $\left[N_{-k}\right]_{j}^{Y_{o}}=0$ unless $j \geq k-2$. Therefore, by (4.18), $B_{m}=0$ unless $m \geq k$.

To prove that the remaining coefficients $B_{m}=0$, we note that by (4.8), in order for the limiting grading $Y_{\infty}$ to exist, we must have

$$
e^{-i a d N_{0}} B_{j} \in \bigoplus_{\ell \geq j+2} E_{\ell}^{Y_{o}}
$$

for any non-zero coefficient $B_{j}$, and hence

$$
B_{j} \in \bigoplus_{\ell \geq j+2} E_{\ell}^{Z}
$$

since $e^{i a d N_{0}}$ is an isomorphism from $E_{\ell}^{Y_{o}}$ to $E_{\ell}^{Z}$.

Corollary. $B_{j}=0$ if $j \geq d$.

To dispense with the remaining coefficients

$$
B_{k}, \ldots, B_{d-2}
$$

we will use that, in the present context,

$$
\left[\frac{\sin N_{0}}{N_{0}} E_{a}^{Y_{o}}\right]_{b}^{Z} \neq 0
$$

iff both $a$ and $b$ are weights and either $a$ is a highest weight, or $a<|b|$. This will be proved in (4.23) below.

To see that all of the coefficients in (4.22) must then vanish, note that by (4.18), such a coefficient $B_{j}$ could be non-zero if and only if:

$$
\left[N_{-k}\right]_{j-2}^{Y_{o}} \neq 0
$$

If $d$ was bigger than $d-2,(4.23)$ would imply

$$
\left[B_{j}\right]_{j}^{Z} \neq 0
$$


on account of (4.18). As this contradicts (4.21), all the coefficients listed in (4.22) must vanish. Accordingly, $N_{-k}$ must be of exactly highest weight $k-2$.

REMARK. In the case where $U$ is of type $E(p, q) \otimes S(n)$ the same analysis applies up until equation (4.18). At this point, one has to further decompose $U=U^{\prime} \oplus U^{\prime \prime}$ as in (4.13). Consequently, on $U^{\prime} k$ gets replaced by $k-\delta$ in equations (4.18) and following. Likewise, on $U^{\prime \prime} k$ is replaced by $k+\delta$. Since $N_{-k}$ is real and $U^{\prime}$ and $U^{\prime \prime}$ are complex conjugates, it then follows from the previous corollary that $N_{-k}$ must be of highest weight $k-\delta-2$ and $k+\delta-2$. Therefore, $\delta=p-q=0$, contradicting the assumption that $p \neq q$.

It only remains to prove

LEMmA 4.23. Let $(\rho, V)$ be a finite-dimensional representation of $s_{2}(\mathbb{C})$,

$$
Y=\rho\left(\begin{array}{cc}
1 & 0 \\
0 & -1
\end{array}\right), \quad Z=\rho\left(\begin{array}{cc}
0 & -i \\
i & 0
\end{array}\right), \quad N=\rho\left(\begin{array}{ll}
0 & 0 \\
1 & 0
\end{array}\right),
$$

$y_{a}, z_{a}$, the natural projections to the a-weight spaces of $Y$ and $Z$, respectively, and

$$
T=\frac{\sin N}{N}=\sum_{n \geq 0} \frac{(-1)^{n}}{(2 n+1) !} N^{2 n} \in \operatorname{End}(V)
$$

Then

$$
z_{b} T y_{a} \neq 0
$$

iff both $a$ and $b$ are weights, and either $a$ is a highest weight or $a<|b|$.

Proof. Can take $\rho$ irreducible of highest weight $d$ and realized on the space of homogeneous polynomials of degree $d$ in two real variables $u, v$. The elements $u^{p} v^{d-p}$ form a basis adapted to $\left(n_{o}, h, n_{o}^{+}\right)$, the elements $w^{p} \bar{w}^{d-p}(w=u+i v)$ form a basis adapted to $\left(x^{-}, z, x^{+}\right)$and $N$ acts by $v \frac{\partial}{\partial u}$. Since

$$
\begin{aligned}
e^{i v \frac{\partial}{\partial u}} u^{p} & =\sum \frac{1}{m !}\left(\frac{\partial}{\partial u}\right)^{m} u^{p}(i v)^{m}=\sum \frac{p(p-1) \cdots(p-m+1)}{m !} u^{p-m}(i v)^{m} \\
& =(u+i v)^{p}=w^{p}
\end{aligned}
$$

one has

$$
\begin{aligned}
e^{i v \frac{\partial}{\partial u}} u^{p} v^{d-p} & =w^{p} v^{d-p}=\frac{1}{(2 i)^{d-p}} w^{p}(w-\bar{w})^{d-p}, \\
e^{-i v \frac{\partial}{\partial u}} u^{p} v^{d-p} & =\frac{1}{(2 i)^{d-p}} \bar{w}^{p}(w-\bar{w})^{d-p}
\end{aligned}
$$

Therefore

$$
\begin{aligned}
T u^{p} v^{d-p} & =\frac{\sin N}{N} u^{p} v^{d-p} \\
& =(2 i)^{-1}\left(e^{i v \frac{\partial}{\partial u}}-e^{-i v \frac{\partial}{\partial u}}\right)\left(v \frac{\partial}{\partial u}\right)^{-1} u^{p} v^{d-p} \\
& =(2 i)^{-1}\left(e^{i v \frac{\partial}{\partial u}}-e^{-i v \frac{\partial}{\partial u}}\right) \frac{1}{p+1} u^{p+1} v^{d-p-1} \\
& =\frac{1}{(2 i)^{d-p+1}(p+1)}\left(w^{p+1}-\bar{w}^{p+1}\right)(w-\bar{w})^{d-p-1}
\end{aligned}
$$


For $p=d$ one gets

$$
T u^{d}=\frac{1}{2 i(d+1)}\left(w^{d+1}-\bar{w}^{d+1}\right)(w-\bar{w})^{-1}=\frac{1}{2 i(d+1)} \sum_{p=0}^{d} w^{p} \bar{w}^{d-p}
$$

which has non-zero projections on all $E_{j}^{Z}$ 's. On the other hand, if $p<d$, then $d-p-1 \geq 0$ and

$$
\begin{aligned}
(2 i)^{d-p+1}(p+1) T u^{p} v^{d-p} & =w^{p+1}(w-\bar{w})^{d-p-1}-\bar{w}^{p+1}(w-\bar{w})^{d-p-1} \\
& =\sum_{j=0}^{d-p-1}\left(\begin{array}{c}
d-p-1 \\
j
\end{array}\right)\left(w^{d-j} \bar{w}^{j}-w^{j} \bar{w}^{d-j}\right)
\end{aligned}
$$

which has non-zero components exactly in

$$
\left(E_{d}^{Z} \oplus \cdots \oplus E_{2 p-d+2}^{Z}\right) \oplus\left(E_{-(2 p-d+2)}^{Z} \oplus \cdots \oplus E_{-d}^{Z}\right) .
$$

This finishes the proof of the Lemma.

5. Split Variations. Here we will prove the following version of Theorem III:

ThEOREM 5.1. Let $\mathcal{V} \rightarrow \Delta^{*}$ be a variation of graded-polarized mixed Hodge structure with unipotent monodromy, and $\mathcal{Y}$ denote the $C^{\infty}$ grading the weight filtration $\mathcal{W}$ obtained by applying (1.2) to each fiber of $\mathcal{V}$. Then, the variation $\mathcal{V}$ is admissible and split if and only if

(a) $\mathcal{Y}$ extends continuously to a grading of $\mathcal{W}$ in the canonical prolongation of $\mathcal{V}$, with limiting value $\lim _{s \rightarrow 0} \mathcal{Y}(s)$ defined over $\mathbb{R}$.

(b) Each of the induced variations $\mathcal{F} G r_{k}^{\mathcal{W}}$ is split.

As noted in the introduction, in order to make the various reality conditions well defined, one must select a non-zero reference element $\theta \in T_{0}(\Delta)^{*}$.

First, the limiting Hodge filtration $F_{\infty}$ of such a variation always exists. If $\mathcal{V}$ is admissible, this is true by definition. On the other hand, if (a) and (b) hold, then $F_{\infty}$ is obtained by extending the graded limiting Hodge filtrations to $V$ via $Y_{\infty}$, since $\mathcal{Y}$ preserves $\mathcal{F}$. More explicitly, if $F: U \rightarrow \mathcal{M}$ represents the period map of $\mathcal{V}$ then

$$
F_{\infty}=\lim _{s \rightarrow 0} \psi(s)
$$

where $\psi(s): \Delta^{*} \rightarrow \check{\mathcal{M}}$ is the map associated to the periodic function

$$
\psi(z)=e^{-z N} \cdot F(z)
$$

Likewise, the hypothesis assert the existence of the grading

$$
Y_{\infty}=\lim _{\operatorname{Im}(z) \rightarrow \infty} Y(z)=\lim _{s \rightarrow 0} Y(s)
$$

where $Y(s)$ is the grading of $W$ defined by the periodic function

$$
Y(z)=e^{-z N} \cdot Y_{(F(z), W)}
$$

Since the graded variation is a sum of variations of pure Hodge structures,

$$
\lim _{s \rightarrow 0} \psi(s) G r^{W}
$$


exists. Since $Y(s)$ preserves the filtration $\psi(s)$, it follows that whenever $Y_{\infty}$ exists, so does $F_{\infty}$. We may therefore write

$$
F(z)=e^{z N} e^{\Gamma(s)} \cdot F_{\infty}, \quad \Gamma(0)=0
$$

relative to a suitable holomorphic function $\Gamma(s)$ (cf. (2.3)).

To complete the proof, we will show that under the hypothesis of the Theorem,

$$
\lim _{\operatorname{Im}(z) \rightarrow \infty} e^{-z N} \cdot Y_{(F(z), W)}=\lim _{\operatorname{Im}(z) \rightarrow \infty} e^{-z N} \cdot Y_{\left(e^{z N} \cdot F_{\infty}, W\right)}
$$

Explanation: if (5.5) holds and $\mathcal{V}$ is admissible and split, $Y_{s}$ extends as described in the introduction. Conversely, if both (5.5) and the hypothesis of the Theorem hold, we can invoke (4.1) to prove that $\mathcal{V}$ is admissible, with limiting mixed Hodge structure $\left(F_{\infty},{ }^{r} W\right)$ which splits over $\mathbb{R}$.

Let $\mathcal{C}$ to be the class of real-analytic functions $q(z)$ which take values in $G L(V)^{W}$ and satisfy an estimate of the form

$$
q(z)=1+o\left(e^{-k y}\right), \quad k>0
$$

for $\operatorname{Im}(z)>>0$, and denote membership in $\mathcal{C}$ by the shorthand $q \approx 1$. The correspondence

$$
(F, W) \leftrightarrow\left(Y_{(F, W)}, F G r^{W}\right)
$$

is (real) birational and smooth along $\mathcal{M}$, since the $I^{p, q}$ are obtained from $F$ and $W$ by taking finite intersections and sums. Consequently, an $\mathcal{M}$-valued function $\phi(z)$ is of the form

$$
\phi(z)=q(z) \cdot F_{o}
$$

relative to a fixed base point $F_{o} \in \mathcal{M}$ and some function $q(z)$ of class $\mathcal{C}$ iff

$$
\left(Y_{(\phi(z), W)}, \phi(z) G r^{W}\right) \rightarrow\left(Y_{\left(F_{o}, W\right)}, F_{o} G r^{W}\right)
$$

exponentially fast. By smoothness, this conclusion remains valid for variable $F_{o}$, provided the limiting value of $F_{o}$ belongs to $\mathcal{M}$. Recall the notation $Y_{F}=Y_{(F, W)}$ for $F \in \mathcal{M}$.

THEOREM 5.6. Let $F(z)$ be the period map of a split admissible variation of graded-polarized mixed Hodge structure with unipotent monodromy. Then, the limiting grading (5.2) may be computed in terms of the corresponding nilpotent orbit, i.e.

$$
\lim _{\operatorname{Im}(z) \rightarrow \infty} e^{-z N} \cdot Y_{F(z)}=\lim _{\operatorname{Im}(z) \rightarrow \infty} e^{-z N} \cdot Y_{e^{z N}} \cdot F_{\infty}
$$

Proof. By (5.3), we may write our period map $F(z)$ in the form

$$
F(z)=e^{z N} q(z) \cdot F_{\infty}
$$

relative to a function $q(z) \approx 1$. Moreover, the splitting ${ }^{r} Y$ of $\left(F_{\infty},{ }^{r} W\right)$ preserves $F_{\infty}$ and satisfies

$$
\left[{ }^{r} Y, N\right]=-2 N
$$

Consequently,

$$
e^{i y N} \cdot F_{\infty}=y^{-\frac{1}{2} r} Y \cdot e^{i N} F_{\infty}
$$


Therefore, upon writing $q_{1}(z)=A d\left(e^{i y N}\right) q(z)$ and $q_{2}(z)=y^{\frac{1}{2}^{r} Y} \cdot q_{1}(z)$, we obtain

$$
\begin{aligned}
F(z) & =e^{x N} e^{i y N} q(z) \cdot F_{\infty}=e^{x N} q_{1}(z) e^{i y N} \cdot F_{\infty} \\
& =e^{x N} q_{1}(z) y^{-\frac{1}{2}^{r} Y} e^{i N} \cdot F_{\infty}=e^{x N} y^{-\frac{1}{2}^{r} Y} q_{2}(z) e^{i N} \cdot F_{\infty}
\end{aligned}
$$

Since $e^{i y N}$ and $y^{-\frac{1}{2}^{r} Y}$ preserve $W$ and are polynomials in positive and negative powers of $y^{\frac{1}{2}}, q_{1}$ and $q_{2} \approx 1$. Since $e^{i N} . F_{\infty} \in \mathcal{M}$ by virtue of Schmid's $S L_{2}$ Orbit Theorem:

$$
Y_{F(z)}=Y_{e^{x N} y^{-\frac{1}{2} r}{ }_{q_{2}}(z) e^{i N} \cdot F_{\infty}}=e^{x N} y^{-\frac{1}{2}^{r} Y} \cdot Y_{q_{2}(z) e^{i N} \cdot F_{\infty}}
$$

The map

$$
\mathcal{M} \rightarrow Y(W), \quad F \in \mathcal{M} \mapsto Y_{(F, W)}
$$

into the space $Y(W)$ consisting of all gradings $Y$ of $W$ is real-algebraic. Since $q_{2} \approx 1$, $e^{i N} . F_{\infty} \in \mathcal{M}$ and $G_{\mathbb{C}}$ acts transitively on $Y(W)$, we conclude:

$$
Y_{q_{2}(z) e^{i N} \cdot F_{\infty}}=q_{3}(z) \cdot Y_{e^{i N}} \cdot F_{\infty}
$$

for some $q_{3} \approx 1$. Consequently, (5.7) implies that

$$
\begin{aligned}
e^{-z N} \cdot Y_{F(z)} & =e^{-i y N} y^{-\frac{1}{2}^{r} Y} q_{3}(z) \cdot Y_{e^{i N} F_{\infty}} \\
& =q_{4}(z) e^{-i y N} y^{-\frac{1}{2} r} Y \cdot Y_{e^{i N}} \cdot F_{\infty}=q_{4}(z) e^{-i y N} \cdot Y_{e^{i y N} F_{\infty}}
\end{aligned}
$$

for some $q_{4} \approx 1$, and hence:

$$
\lim _{\operatorname{Im}(z) \rightarrow \infty} e^{-z N} \cdot Y_{F(z)}=\lim _{\operatorname{Im}(z) \rightarrow \infty} e^{-z N} \cdot Y_{e^{z N}} \cdot F_{\infty}
$$

COROLlARY. Under the hypothesis of Theorem (5.6), the period map $F(z)$ satisfies $(a)$ and $(b)$. Moreover, in this setting the limiting grading $Y_{\infty}$ coincides with the grading of $W$ obtained by applying the first theorem of the Appendix to the pair

$$
\left(N,{ }^{r} Y\right), \quad{ }^{r} Y=Y_{\left(F_{\infty}, r W\right)}
$$

To prove the converse, let $\rho$ be the representation of $s l_{2}(\mathbb{R})$ attached to a variation $\mathcal{V}$ which satisfies (a) and (b) of Theorem (5.1), i.e. pull back the representations $\rho_{k}$ which define the associated nilpotent orbits $e^{z N} . F G r_{k}^{W}$-which are $S L_{2}$ Orbits, on account of (b), via the grading $Y_{\infty}$, which is defined over $\mathbb{R}$. Then, as in $\S 4$ :

(1) $N_{0}=\rho(h)$, where

$$
N=N_{0}+N_{-1}+\cdots
$$

denotes the decomposition of $N$ with respect to the eigenvalues of $a d Y_{\infty}$.

(2) $Y_{o}=\rho(h)$ preserves $F_{\infty}$.

Likewise, conditions (a) and (b) imply that $Y_{\infty}$ preserves $F_{\infty}$, since the grading $Y(s)$ from (5.2) preserves the filtration $\psi(s)$.

THEOREM 5.8. Let $F(z)$ be the period map of a variation of graded-polarized mixed Hodge structure with unipotent monodromy for which conditions (a) and (b) of Theorem (5.1) hold. Then,

$$
Y_{\infty}=\lim _{\operatorname{Im}(z) \rightarrow \infty} e^{-z N} \cdot Y_{e^{z N}} \cdot F_{\infty}
$$


Proof. By (5.3),

$$
F(z)=e^{z N} g(z) \cdot F_{\infty}
$$

for some function $g(z) \approx 1$. Since $\mathcal{C}$ is closed under conjugation by $e^{z N}$,

$$
F(z)=q(z) e^{z N} \cdot F_{\infty}
$$

for some function $q \approx 1$. Next, we prove that for a suitable integer $m \in \mathbb{Z}$, the endomorphism

$$
T=Y_{o}+m Y_{\infty}, \quad Y_{o}=\rho(H)
$$

satisfies the estimate

$$
y A d\left(y^{T}\right) N=N_{0}+o\left(y^{-1}\right)
$$

Indeed, decompose $N=N_{0}+N_{-1}+\cdots$ according to the eigenvalues of $Y_{\infty}$. Then,

$$
y A d\left(y^{T}\right) N=A d\left(y^{\frac{1}{2} Y_{o}}\right) y A d\left(y^{m Y_{\infty}}\right) N=N_{0}+\sum_{j \geq 1} y^{1-m j} A d\left(y^{\frac{1}{2} Y_{o}}\right) N_{-j} .
$$

Since $j \geq 1$ and only finitely many eigenvalues of $Y_{o}$ appear non-trivially in each $N_{j}$, we may force the leftmost sum to involve only negative powers of $y$, by making $m$ sufficiently large.

By hypothesis, $T$ is defined over $\mathbb{R}$. The remarks immediately preceding (5.8), $T$ also preserves $F_{\infty}$. Hence

$$
e^{i y N} \cdot F_{\infty}=e^{i y N} y^{-T} \cdot F_{\infty}=y^{-T} e^{i y A d\left(y^{T}\right) N} \cdot F_{\infty}=y^{-T} P(y) e^{i N_{0}} \cdot F_{\infty}
$$

relative to a function $P(y)$ which is polynomial in $y^{-1}$.

To finish the proof, we note that by virtue of $(b)$ and the $S L_{2}$ Orbit Theorem, $e^{i N_{0}} \cdot F_{\infty} \in \mathcal{M}$. Since $\mathcal{C}$ is closed under conjugation by $y^{k T}, k \in \mathbb{R}$ :

$$
\begin{aligned}
F(i y) & =q(i y) e^{i y N} \cdot F_{\infty}=q(i y) y^{-T} e^{i y A d\left(y^{T}\right) N} \cdot F_{\infty} \\
& =y^{-T} q_{1}(y) e^{i y A d\left(y^{T}\right) N} \cdot F_{\infty}
\end{aligned}
$$

and hence

$$
Y_{F(i y)}=y^{-T} \cdot Y_{q_{1}(i y) e^{i y A d\left(y^{T}\right) N} \cdot F_{\infty}}=y^{-T} q_{2}(y) \cdot Y_{e^{i y A d\left(y^{T}\right) N} \cdot F_{\infty}} .
$$

Accordingly,

$$
\begin{aligned}
& e^{-i y N} . Y_{F(i y)}=e^{-i y N} y^{-T} q_{2}(y) \cdot Y_{e^{i y A d\left(y^{T}\right) N} \cdot F_{\infty}}=q_{3}(y) e^{-i y N} y^{-T} \cdot Y_{e^{i y A d\left(y^{T}\right) N} \cdot F_{\infty}} \\
& =q_{3}(y) e^{-i y N} \cdot Y_{y^{-T}} e^{i y A d\left(y^{T}\right) N} \cdot F_{\infty}=q_{3}(y) e^{-i y N} \cdot Y_{e^{i y N} y^{-T}} \cdot F_{\infty} \\
& =q_{3}(y) e^{-i y N} \cdot Y_{e^{i y N} . F_{\infty}}
\end{aligned}
$$

for functions $q_{2}$ and $q_{3} \approx 1$, where from

$$
\lim _{y \rightarrow \infty} e^{-i y N} \cdot Y_{e^{i y N}} . F_{\infty}=Y_{\infty}
$$

Appendix. Two theorems of Deligne [8]. Let $0 \subseteq \cdots \subseteq W_{k} \subseteq W_{k+1} \subseteq$ $\cdots \subseteq V$ be an increasing filtration of a finite dimensional vector space $V$ defined over an algebraically closed field $k$ of characteristic zero. 
THEOREM 1. Let $N$ be a nilpotent endomorphism of $V$ for which the corresponding relative weight filtration ${ }^{r} W={ }^{r} W(N, W)$ exists, and ${ }^{r} Y$ be a grading of ${ }^{r} W$ which preserves $W$ and satisfies the additional condition $\left[{ }^{r} Y, N\right]=-2 N$. Then, there exists a unique grading $Y$ of $W$ such that

(1) $\left[{ }^{r} Y, Y\right]=0$.

(2) $N=N_{0}+N_{-2}+\cdots$ when decomposed relative to the eigenvalues of ad $Y$.

(3) $\left(a d N_{0}\right)^{k-1} N_{-k}=0$ for all $k>0$.

Sketch of Proof. The desired grading $Y$ may be constructed as follows: Let $Y_{o}$ be a grading of $W$ for which $\left[{ }^{r} Y, Y\right]=0$, and note that the group

$$
G_{o}=\left\{g \in G L(V) \mid\left[g,{ }^{r} Y\right]=0, \quad(g-1): W_{k} \rightarrow W_{k-1} \forall k\right\}
$$

acts transitively on the set of all such gradings. Assume by induction that the initial grading $Y_{o}$ satisfies (1)-(3) modulo the ideal

$$
\operatorname{Lie}_{-r}=\left\{\alpha \in \operatorname{End}(V) \mid \alpha: W_{k} \rightarrow W_{k-r} \forall k\right\}
$$

Then one may construct an element $g \in G_{o}$ such that, relative to the grading $Y^{\prime}=$ $A d(g) Y_{o}$, (1)-(3) hold modulo the ideal Lie $_{-(r+1)} \subseteq$ Lie $_{-r}$. Since Lie $e_{-r}=0$ for some finite index $r$, the desired grading $Y$ will be obtained after finitely many steps. The details are discussed in [8][18].

REMARK. The preceding result may be reformulated as the statement that given the existence of ${ }^{r} W={ }^{r} W(N, W)$ and a grading ${ }^{r} Y$ of the type described above, there exists a unique grading $Y$ of $W$ which commutes with ${ }^{r} Y$ and has the property that the associated $s l_{2}$-triple $\left(N_{0},{ }^{r} Y-Y, N_{0}^{+}\right)$satisfies the commutativity condition $\left[N-N_{0}, N_{0}^{+}\right]=0$.

LEMMA. The construction of Theorem 1 is both functorial and compatible with the operations of direct sum, tensor product and dual.

To state Deligne's second theorem, we begin with a triple $(F, W, N)$ of the type arising from the degeneration of an admissible variation of graded-polarizable mixed Hodge structure, i.e.

$-V=V_{\mathbb{R}} \otimes \mathbb{C}$ relative to some underlying real form $V_{\mathbb{R}}$ to which both $W$ and $N$ descend.

- The relative weight filtration ${ }^{r} W={ }^{r} W(N, W)$ exists.

- The pair $\left(F,{ }^{r} W\right)$ is a mixed Hodge structure relative to which $N$ is a $(-1,-1)$-morphism and $W$ is a filtration by sub-mixed Hodge structures.

In particular, given any such triple $(F, W, N)$, we may construct an associated grading

$$
Y=Y(F, W, N)
$$

of $W$ via application of Theorem 1 to the grading ${ }^{r} Y$ of ${ }^{r} W$ determined by the $I^{p, q}$ 's of the mixed Hodge structure $\left(F,{ }^{r} W\right)$.

REMARK. Inspection of the proof of Theorem 1 shows that whenever the mixed Hodge structure $\left(F,{ }^{r} W\right)$ associated to the triple $(F, W, N)$ is split, the corresponding grading $Y=Y(F, W, N)$ lies in $\operatorname{End}\left(V_{\mathbb{R}}\right)$. 
Lemma. The grading $Y=Y(F, W, N)$ constructed above preserves $F$.

Sketch of Proof. (cf. [18] for details). One uses the preceding lemma to reduce the problem to the case where $\left(F,{ }^{r} W\right)$ is a split mixed Hodge structure which is either of the form

- $V=\oplus_{p} I^{p, p}$ with $N\left(I^{p, p}\right) \subseteq I^{p-1, p-1}$ or,

$-V=I^{p, 0} \oplus I^{0, p}$ and $N=0$.

TheOREM 2. Let $(F, W, N)$ be a triple of the type described above for which the mixed Hodge structure $\left(F,{ }^{r} W\right)$ is split. Then, the pair $\left(e^{i y N} . F,{ }^{r} W\right)$ defines a mixed Hodge structure for all $y>0$. Moreover, the grading $Y_{\left(e^{i y N} . F, W\right)}$ of $W$ determined by the $I^{p, q}$ 's of $\left(e^{i y N} . F, W\right)$ is given by the formula

$$
Y_{\left(e^{i y N} . F, W\right)}=e^{i y N} . Y(F, W, N)
$$

Proof. As a consequence of [5], the pair $\left(e^{i y N} . F, W\right)$ is a mixed Hodge structure for all $y>0$. To establish the stated formula, we define $Y$ to be $Y(F, W, N)$, and note that

$$
Y=Y_{\left(e^{i y N_{0}} . F, W\right)}
$$

for all $y>0$ since, in the present context, $Y$ is a grading of $W$ which is both defined over $\mathbb{R}$ and preserves $e^{i y N_{0}} . F$. Accordingly, in order to establish Theorem 2, it will suffice to show that

$$
e^{\phi(y)}:=e^{i y N} e^{-i y N_{0}} \in \exp \left(\Lambda_{\left(e^{\left.i y N_{0} . F, W\right)}\right.}^{-1,-1}\right)
$$

Indeed, if the preceding equation is true, then

$$
\begin{aligned}
Y_{\left(e^{i y N} . F, W\right)} & =Y_{\left(e^{i y N} e^{-i y N_{0}} e^{i y N_{0}} . F, W\right)} \\
& =e^{i y N} e^{-i y N_{0}} \cdot Y_{\left(e^{i y N_{0}} . F, W\right)}=e^{i y N} e^{-i y N_{0}} \cdot Y=e^{i y N} \cdot Y
\end{aligned}
$$

To verify $(*)$, note that $\phi(y)$ is a Lie polynomial in the monomials $\left(a d N_{0}\right)^{a} N_{-k}$. In particular $\phi(y)$ contains no $N_{0}$ term. Moreover, by conditions (2) and (3) of Theorem $1,\left(a d N_{0}\right)^{a} N_{-k}$ is zero unless $k>1$ and $0 \leq a \leq k-2$. It then follows from (4.12) that

$$
\left(a d N_{0}\right)^{a} N_{-k} \in \bigoplus_{0<r<k} g l(V)_{\left(e^{\left.i y N_{0}, W\right)}\right.}^{-r, r-k} \subseteq \Lambda_{\left(e^{i y N_{0} . F, W}\right)}^{-1,-1}
$$

Consequently, $\phi(y)$ takes values in $\Lambda_{\left(e^{i y N_{0}}, F, W\right)}^{-1,-1}$ since the latter is a Lie subalgebra of $g l(V)$.

\section{REFERENCES}

[1] Biquard O., Fibrés de Higgs et connexions intégrables: le cas logarithmique (diviseur lisse), Ann. Sci. École Norm. Sup. (4), 30:1 (1997), pp. 41-96.

[2] Biquard O. AND BoAlch P., Wild non-abelian Hodge theory on curves, math.DG/0111098 (2002), to appear in Compositio Math..

[3] Bloch S. And ESNAult H., Homology for irregular connections, math.AG/0005137v1 (2000), to appear in Proc. of Luminy Conference on Conductors.

[4] Brylinski J. and Zucker S., An Overview of Recent Advances in Hodge Theory, in Complex Manifolds, (Gamkrelidze R. ed.), Springer, 1997. 
[5] Cattani E., Deligne P. And Kaplan A., On the locus of Hodge cycles, Journal Amer. Math. Soc., 8 (1995), pp. 483-506.

[6] Cattani E., Kaplan A. And Schmid W., Degeneration of Hodge structures, Ann. of Math., 123 (1986), pp. 457-535.

[7] Cattani E., Kaplan A. And Schmid W., $L_{2}$ and Intersection cohomologies with coefficients in a variation of Hodge structure, Invent. Math., 87 (1986), pp. 217-252.

[8] Deligne P., Private communication (1993).

[9] — Appendix to Variation of Mixed Hodge Structure I, Invent. Math., 80 (1985), pp. 489 -542 .

[10] — La conjecture de Weil II, Publ. Math. IHES, 52 (1980), pp. 137-252.

[11] — Equations différentielles à points singuliers réguliers, in Lecture Notes in Mathematics $163,1970$.

[12] Griffiths P., Periods of integrals on algebraic manifolds, Bull. Amer. Math. Soc., 76 (1970), pp. 228-296.

[13] HaIn R. AND ZuCker S., Unipotent variations of mixed Hodge structure, Invent. Math., 88 (1987), pp. 83-124.

[14] KAPLAN A., Notes on the moduli spaces of Hodge structures, Unpublished manuscript (1995).

[15] Martinet J. And RAmis J.-P., Elementary acceleration and multisummability, Ann. Inst. H. Poncare Phys. Theor., 54:4 (1991), pp. 331-401.

[16] Matsumoto M., Arithmetic fundamental groups and moduli of curves, Lect. Notes Abdus Salam Int. Cent. Theoret. Phys., 1 (2000), pp. 355-383.

[17] Pearlstein G., Variations of Mixed Hodge Structure, Higgs Fields, Quantum Cohomology, Manuscripta Math., 102 (2000), pp. 269-310.

[18] Pearlstein G., Degenerations of Mixed Hodge Structure, Duke Math. J., 110 (2001), pp. 217251.

[19] Pearlstein G., $S L_{2}$ Orbits and Degenerations of Mixed Hodge Structure, in math.AG/0309439.

[20] Schmid W., Variation of Hodge Structure: The Singularities of the Period Mapping, Invent. Math., 22 (1973), pp. 211-319.

[21] Simpson C., Harmonic bundles on noncompact curves, J. Amer. Math. Soc., 3:3 (1990), pp. 713770.

[22] Steenbrink J., Limits of Hodge structure, Invent. Math., 31 (1976), pp. 229-257.

[23] Steenbrink J. And Zucker S., Variation of Mixed Hodge Structure I, Invent. Math., 80 (1985), pp. 489-542. 